\title{
Temporary book-tax differences under IFRS
}

This research seeks to determine whether the general and specific categories of deferred tax assets (DTAs) and deferred tax liabilities (DTLs) under International Financial Reporting Standards are related to changes in stock prices. The results seem to indicate that DTAs and DTLs under IFRS could be less helpful to investors than they currently are under US GAAP.

Keywords: deferred tax assets, deferred tax liabilities, IFRS, stock prices.

Tyrimu siekiama nustatyti ar bendrosios ir specifinès atidètojo pelno mokesčio turto (APMT) ir atidètojo pelno mokesčio įsipareigojimų (APMİ) kategorijos turi sąsajų su akcijų kainų pokyčiais, remiantis Tarptautinès finansinés atskaitomybès standartais (TFAS). Tyrimo rezultatai parodé, kad remiantis APMT ir APMI akciju kainų pokyčių prognozavimas yra kur kas ribotesnis, lyginant su JAV, kur naudojami bendrieji atskaitomybès principai (angl. GAAP).

Raktiniai žodžiai: atidètojo pelno mokesčio turtas, atidèto pelno mokesčio įsipareigojimai, Tarptautiniai finansinès atskaitomybės standartai (TFAS), vertybinių popierių kainos.

JEL Classification: M40.

\section{Introduction}

Objective. Deferred tax assets (DTAs) and deferred tax liabilities (DTLs) disclosures many times more accurately predict future stock price changes than earnings per share, net change in assets, or cash flows. As such, investors, creditors, regulators, and other financial statement users must learn how these DTAs and DTLs predict these future stock price changes. However, despite significant extant research discussing the effect of singular or general categories of DTAs or DTLs on stock prices, no research has considered all the categories simultaneously. Furthermore, no extant research demonstrates the relationships between DTAs and DTLs on the one hand and stock prices on the other hand under International Financial Reporting Standards (IFRS). Thus, this paper, for the first time, seeks to ascertain which of the specific differences between temporary book income and taxable income (BTDs) provide stock price predicting value under IFRS. These specific differences are especially important for discussion as the world moves toward exclusive use of IFRS. Many countries already have implemented IFRS without background knowledge on the significant power of BTDs under these standards. This research is also still of great importance to non-implementing countries, such as the US, as it can inform 
regulators and legislators of the incremental value that temporary BTDs could provide under IFRS to compare to already extant research on temporary BTDs under their current standards.

Tasks. The BTDs are hand collected from the Financial Times and Stock Exchange (FTSE) 100's annual reports for the years 2004 through 2010. Then, correlations and logistic regressions are done to determine the statistical significance of these variables. J. Thomas and F. Zhang (2007) utilized these methodologies. B. Lev and D. Nissim (2004) did as well.

Research methods. This work involves synthesizing scientific literature, evaluating annual reports, comparing results, and disclosing the findings here. The previous discussion of the tasks provides more detail on the implementation of the research method. The previous discussion of the targets establishes the samples for these research methods.

Research results. Until recently, no researcher had even tried to review US Generally Accepted Accounting Principles (GAAP) DTAs and DTLs disclosures to determine which specific categories within DTAs and DTLs were more powerful than the others. Even this research is so new that it is not published yet. The reason for this lack of inquiry owes to the lack of computer-readable information on tax footnotes. The first challenge to the power of BTDs normally emerges from the fact that other footnote disclosures contain information on some of those temporary differences. However, in due course in the following pages, this challenge is resolved. The research at hand involves reviewing the footnote disclosures on BTDs for the FTSE 100 from 2004 through 2010, the implementation of IFRS to the current date. These data provide the basis for testing stock returns with specific BTDs.

Researchers have demonstrated the influence of BTDs with regard to many issues (Graham et al., 2010). Research has shown the relationships between BTDs and each of the following: current and future stock returns, earnings growth, financing costs, and price-to-earnings ratios, etc. (Graham et al., 2010).

The significance of BTDs is readily understandable on consideration of the fact that BTDs deal with converging financial statements and tax returns. This situation in of itself means that most company activities move through the DTAs and DTLs at some timely point. The real question then becomes which temporary differences are most significant in the IFRS context.

The start of the process involves investigating which specific temporary differences are informative. Next, long-window returns are regressed on the remaining BTDs.

Some BTDs provide information showing DTAs and DTLs as significant to stock price. Because not all temporary BTDs provide information, some people still could reject the general proposition that BTDs establish highly relevant information to investors in setting stock prices. The paper continues in the following order: literature review, methodology, sample, results, conclusions, and implications.

\section{Literature review}

DTAs and DTLs under US GAAP. Many researchers have demonstrated connections between DTAs and DTLs on the one hand and stock price reactions on the other hand, such as cataloged in J. L. Blouin et al. (2004) and J. R. Graham et al. (2010). Nevertheless, 
none has explored the topic as comprehensively or in the context of IFRS as this research project does. This initial line of research began with W. Beaver and R. Dukes (1972), who established the incremental value of deferred tax components over earnings per share data. J. Rayburn (1986) continued the line, showing that tax accruals are more correlated with stock price changes than cash flows. P. Chaney and D. Jeter (1994) essentially reinforced W. Beaver and R. Dukes (1972). E. Amir and M. Kirschenheiter (1997), E. Amir and T. Sougiannis (1999), B. Ayers (1998), and D. Dhaliwal et al. (2000) then followed but failed to pursue the issues as comprehensively as this research paper does.

Unlike this current research that explores the influence of specific categories on stock prices, E. Amir and M. Kirschenheiter (1997) explored which general categories of DTAs and DTLs temporary differences influence stock prices. They found that the market discounts DTAs and DTLs based on their likelihood of and time to reversal.

Different from this current research that explores all categories of DTAs and DTLs, E. Amir and T. Sougiannis (1999) considered but one category of DTAs, specifically the DTAs carryforwards. They discovered that earnings from carryforward companies are less persistent, but the DTAs carryforwards do help investors' predict prices.

Unlike this current research that details the differences from US GAAP discoveries in IFRS, B. Ayers (1998) researched whether the current US GAAP, Financial Accounting Standard (FAS) 109, is more relevant than the previous US GAAP, Accounting Principles Board (APB) 11. FAS 109 has greater relevance to price than APB 11. Supporting this current research, B. Ayers (1998) showed that DTAs and
DTLs have separable effects on price. This discovery supported this current research methodology.

Different from this current research that explores all categories of DTAs and DTLs, D. Dhaliwal et al. (2000) ascertained whether DTLs not reported on the balance sheet are valued in the context of "First In, First Out" (FIFO) or "Last in, First Out" (LIFO) choices. If investors choose to value all companies based on FIFO, it would require an adjustment of LIFO to this other method to compare earnings reports. If inventory costs are increasing globally, then some stock price effect could result from the implied increase to DTLs. Thus, D. Dhaliwal et al. (2000) found that the market does value the DTLs not on the balance sheet.

General tax disclosures' influence on stock prices. Then, further research on the significance of general tax disclosures to stock price changes supports this current research inquiry. Taxable income is the figure reported to tax authorities. Book income is the figure reported to the public in earnings reports. Establishing that tax disclosures do influence stock prices, R. Lipe (1986) found that the general category of income tax expense contained more influential information than other earnings components.

M. Hanlon (2005) reviewed whether taxable income disclosures were more informative than book income. Their results showed that book income has the larger coefficient and $t$-statistic. However, taxable income is statistically significant, which indicates that it provides information that book income alone does not.

B. Lev and D. Nissim (2004) researched the effect of taxable income and book income differences on earnings growth and therein earnings quality. They determined 
that the ratio of book and taxable income provides information that accrued earnings and cash flows do not. Companies choose discretionary accruals for book, not taxable, income disclosures. Reversals reduce the quality of accrued earnings. To the extent that companies seek to have consistent taxable income, taxable income provides information on expected future taxable income. Thus, recording high taxable income currently shows an expectation of high taxable income and therein high book income in subsequent years. Because companies tend to recognize income for tax before the corresponding revenue for book, high ratios should forecast high future book revenues. Because companies tend to recognize deductions for tax after expenses for book, high ratios should forecast low future book expenses.

M. Hanlon (2005) determined that companies with the largest book-tax differences have less persistent earnings, accruals, and cash flows. Where book income is substantially less than estimated taxable income, stock pricing properly imports persistence of earnings and cash flows but overstates accruals' persistence. Where book income is substantially greater than estimated taxable income, stock pricing properly imports accruals' persistence but understates the persistence of earnings and cash flows.

J. Thomas and F. Zhang (2007) looked at the relationship between taxable income and future returns. Unexpected results for estimated taxable income correspond with stock returns six months later. B. Lev and D. Nissim (2004) found it to be one year. J. Thomas and F. Zhang (2007) discussed two reasons for this discovered relationship. The tax surprise could forecast subsequent book income not ascertainable from the current book income numbers. It could also forecast other subsequent financial information.
D. Weber (2009) showed that only for companies with lower-quality information does the relationship between book-to-tax differences and future returns exist. The research found that prediction errors are statistically significant to taxable-to-bookincome ratios. It also disclosed that prediction errors are more positive where large book-to-tax differences exist. J. R. Graham et al. (2010) demonstrated that tax information has an effect on future stock returns and prices.

Despite the importance of establishing that DTAs and DTLs directly and indirectly influence stock prices, no research heretofore has considered which specific components of DTAs and DTLs are significant. Furthermore, as any individual can witness from all the previously discussed research that involves US GAAP, research has not sufficiently dealt with the effects of IFRS tax disclosures on stock prices. This research combines these categories of inquiry to produce results important for fully understanding the significance of IFRS tax disclosures. The following is the first extant research into these areas.

\section{Methodology}

For years, finance professionals have discussed whether the efficient market hypothesis exists. As such, this research pursues the efficient and less than fully efficient market hypotheses separately to show whether DTAs and DTLs are statistically significant under IFRS regardless of the market presumption. The efficient market hypothesis is tested with simultaneous disclosure of the DTAs and DTLs and their instantaneous effect on stock prices. The less than fully efficient market hypothesis is tested with the disclosure of DTAs and DTLs in year 1 
followed with the stock change measured from that date through the end of year 2.

To begin, IFRS' DTAs and DTLs do provide information relevant to stock price. Table 1 and Table 2 provide this information. If the changes in stock prices of the FTSE 100 are regressed on their aggregate DTAs and DTLs under the efficient market hypothesis, these results do show. The aggregate DTAs are strongly explanatory of those price changes [-.162 $(-1.468)]$. The aggregate DTLs are statistically significant to those price changes $\left[.232^{*}(2.103)\right]$. Under the less efficient market model, the aggregate DTAs explain those price changes well $[-.141(-1.263)]$. The aggregate DTLs are not statistically significant but are just outside 10 percent statistical significance [.176 (1.580)]. In each table below, ${ }^{*}$ and ${ }^{* *}$ stand for .05 and .01 statistical significance levels.

Table 1

2004 through 2009 changes in prices regressed on 2004 through 2009 changes in aggregate

\section{DTAs and DTLs}

\begin{tabular}{|l|c|}
\hline Variable & $\mathbf{0 4 - 0 9}$ \\
\hline DTAs & -.162 \\
& $(-1.468)$ \\
\hline DTLs & $\mathbf{. 2 3 2}$ \\
& $(2.103)$ \\
\hline
\end{tabular}

Table 2

2005 through 2010 changes in prices regressed on 2004 through 2009 changes in aggregate DTAs and DTLS

\begin{tabular}{|l|c|}
\hline Variable & $\mathbf{0 5 - 1 0}$ \\
\hline \multirow{2}{*}{ DTAs } & -.141 \\
& $(-1.263)$ \\
\hline \multirow{2}{*}{ DTLs } & .176 \\
& $(1.580)$ \\
\hline
\end{tabular}

With the fact that extant research already demonstrates that DTAs and DTLs can be incrementally explanatory of stock price changes over pretax book income, the following research does not test the results from such an inquiry. Instead, it seeks to determine which parts of the DTAs and DTLs are underlying this previously referenced explanatory power of DTAs and DTLs over stock price changes.

Table 3

2004 through 2005 changes in prices regressed on 2004 through 2005 changes in aggregate DTAs and DTLs

\begin{tabular}{|c|c|c|c|c|c|}
\hline Variable & 04-05 & 05-06 & 06-07 & 07-08 & 08-09 \\
\hline DTAs & $\begin{array}{l}.012 \\
(.119) \\
\end{array}$ & $\begin{array}{l}.099 \\
(.988) \\
\end{array}$ & \begin{tabular}{|l|}
$\mathbf{3 5 8}^{* *}$ \\
$(3.791)$ \\
\end{tabular} & \begin{tabular}{|l|}
-.094 \\
$(-.939)$ \\
\end{tabular} & \begin{tabular}{|l|}
-.043 \\
$(-.424)$ \\
\end{tabular} \\
\hline DTLs & \begin{tabular}{|l|}
-.041 \\
$(-.406)$ \\
\end{tabular} & \begin{tabular}{|l|}
-.023 \\
$(-.229)$ \\
\end{tabular} & $\begin{array}{l}.495^{\star *} \\
(5.645)\end{array}$ & $\begin{array}{l}.279^{\star *} \\
(2.782)\end{array}$ & \begin{tabular}{|l|}
-.007 \\
$(-.072)$
\end{tabular} \\
\hline
\end{tabular}

Table 4

2005 through 2006 Changes in prices regressed on 2004 through 2005 changes in aggregate DTAs and DTLs

\begin{tabular}{|l|r|r|r|r|r|}
\hline Variable & $\mathbf{0 5 - 0 6}$ & $\mathbf{0 6 - 0 7}$ & $\mathbf{0 7 - 0 8}$ & $\mathbf{0 8 - 0 9}$ & $\mathbf{0 9 - 1 0}$ \\
\hline \multirow{2}{*}{ DTAs } & -.117 & .147 & $-.306^{* *}$ & -.078 & -.031 \\
& $(-1.168)$ & $(1.468)$ & $(-3.187)$ & $(-.775)$ & $(-.308)$ \\
\hline \multirow{2}{*}{ DTLs } & -.004 & $\mathbf{. 2 0 5}$ & $-.504^{*}$ & -.142 & -.127 \\
& $(-.043)$ & $(2.071)$ & $(-5.784)$ & $(-1.417)$ & $(-1.263)$ \\
\hline
\end{tabular}

As Table 3 and Table 4 show, the reason for aggregate DTAs and DTLs being explanatory of price could result from year to year differences. The changes in price regressed on the changes in aggregate DTAs and DTLs from 2006 through 2007 and 2007 through 2008 are statistically significant, but the other year to year changes are not. Thus, throughout the following discussion, control is made for year to year changes through reviewing results for each year where possible.

Throughout the course of this research, no presumption is made favoring the efficient market hypothesis over any less 
efficient market notion. The extant research strongly supports the existence of less than fully efficient markets. However, the inclusion of the efficient market results (price changes from 2004 through 2005 compared to DTA and DTL changes from 2004 through 2005) at the least controls somewhat for the results viewed from the less efficient market testing (price changes from 2005 through 2006 compared to DTA and DTL changes from 2004 through 2005).

In fact, Table 5 presents unique patterns to the DTAs and DTLs, which could distort any efficient or less than efficient discussion. The DTAs seem to be related over two-year time periods. At the same time, DTLs do not evidence that pattern. However, the DTLs seem to move through the next year's DTAs.

The 2004-05 DTAs is statistically significant to the 2006-07 DTAs and the 2008-09 DTAs. The 2006-07 DTAs is close to being statistically significant to the 2008-09 DTAs (.148). These items indicate patterns in the DTAs in two-year pairings.

The 2004-05 DTLs is statistically significant with the 2006-07 DTAs. The 2005-06 DTLs is statistically significant with the 2006-07 DTAs. The 2006-07 DTLs is close to being statistically significant with the 2007-08 DTAs (.136). The 2007-08 DTLs is statistically significant to the 2008-09 DTAs. These items indicate patterns in the DTLs somehow passing through the DTAs of the next year.

Separate reviews of the FTSE 100 results under industry category do not show any industry in general to be moving the results. The sample size makes it difficult to determine with absolute certainty whether any industry is responsible for the significance of the results. However, these separate tabulations do disclose that the financial industry has some bearing on the magnitude of DEBT (bad debts) changes. Thus, any findings of statistical significance for this category should be considered in light of this fact. Otherwise, though, there is no necessity to control for industry through any other means, such as indicator variables.

To be completely expressive here then, no clustering or company fixed effects are employed in the subsequent model. However, there should be no issue with regard to understated standard errors as first differences are taken, especially in the surprise measuring research.

\section{Correlations between aggregate DTAs and DTLs}

Table 5

\begin{tabular}{|c|c|c|c|c|c|c|c|c|c|c|}
\hline Variable & $\begin{array}{l}\text { 04-05 } \\
\text { DTAs }\end{array}$ & $\begin{array}{l}\text { 04-05 } \\
\text { DTLs }\end{array}$ & $\begin{array}{l}\text { 05-06 } \\
\text { DTAs }\end{array}$ & $\begin{array}{l}\text { 05-06 } \\
\text { DTLs }\end{array}$ & $\begin{array}{l}\text { 06-07 } \\
\text { DTAs }\end{array}$ & $\begin{array}{l}\text { 06-07 } \\
\text { DTLs }\end{array}$ & $\begin{array}{l}\text { 07-08 } \\
\text { DTAs }\end{array}$ & $\begin{array}{l}\text { 07-08 } \\
\text { DTLS }\end{array}$ & $\begin{array}{l}\text { 08-09 } \\
\text { DTAs }\end{array}$ & $\begin{array}{l}\text { 08-09 } \\
\text { DTLS }\end{array}$ \\
\hline 04-05 DTAs & 1 & & & & & & & & & \\
\hline 04-05 DTLs & -.172 & 1 & & & & & & & & \\
\hline 05-06 DTAs & .049 & .125 & 1 & & & & & & & \\
\hline 05-06 DTLs & $.227^{\star}$ & -.157 & $.492^{\star *}$ & 1 & & & & & & \\
\hline 06-07 DTAs & $.327^{\star *}$ & $-.209^{\star}$ & $.541^{\star *}$ & $.362^{\star \star *}$ & 1 & & & & & \\
\hline 06-07 DTLs & -.043 & -.147 & $.389^{\star *}$ & $.391^{\star *}$ & $.599^{* *}$ & 1 & & & & \\
\hline 07-08 DTAs & .103 & -.171 & -.082 & .145 & $.356^{* *}$ & .136 & 1 & & & \\
\hline 07-08 DTLs & .007 & .190 & -.189 & $-.496^{* *}$ & -.079 & $-.488^{\star \star}$ & -.149 & 1 & & \\
\hline 08-09 DTAs & $.270^{\star *}$ & -.152 & .155 & $.233^{*}$ & .148 & .088 & -.007 & $-.227^{\star}$ & 1 & \\
\hline 08-09 DTLs & -.097 & .059 & $.418^{\star *}$ & $.435^{\star \star}$ & -.030 & .148 & .002 & $-.301^{\star \star}$ & $.450^{\star *}$ & 1 \\
\hline
\end{tabular}


The significance of BTDs to investors could emerge from two factors. BTDs could give investors information as to the quality of financial statements or express further information as to the company's tax liabilities. The BTDs could enhance quality through enabling comparisons between book financial statement numbers and an alternative system of tax return numbers. The extra detail from disaggregated components could also establish the incremental value.

Regression equation. To determine whether specific BTDs are incrementally informative, the following regression equation is utilized:

PRICE $=\alpha+$ DTAs $[\beta 1($ DEPR $)+\beta 2(\mathrm{~EB})$ $+\beta 3(\mathrm{ESOP})+\beta 4(\mathrm{DEBT})+\beta 5(\mathrm{INV})+$ $\beta 6(\mathrm{UNEARN})+\beta 7(\mathrm{DERIV})+\beta 8(\mathrm{INVEST})$ $+\beta 9($ EQUITY $)+\beta 10($ INTAN $)+$ $\beta 11($ ITEMS $)+\beta 12($ LOSSES $)+\beta 13($ RESERV $)$ $+\beta 14($ ACCRUE $)+\beta 15$ (OTHER) $]+$ DTLs $[\beta 16(\mathrm{DEPR})+\beta 17(\mathrm{~EB})+\beta 18(\mathrm{ESOP})+$ $\beta 19($ RECEIV $)+\beta 20($ INV $)+\beta 21($ DEFER $)+$ $\beta 22($ DERIV $)+\beta 23(\mathrm{FV})+\beta 24($ EQUITY $)+$ $\beta 25($ INTAN $)+\beta 26($ LOSSES $)+\beta 27(\mathrm{CONV})$ $+\beta 28(\mathrm{PROV})+\beta 29(\mathrm{ACCRUE})+$ $\beta 30($ OTHER $)]+\epsilon$

The regression equation advances M. Hanlon (2005). However, just as M. Hanlon (2005) does, each year is tested separately. Thus, any categorical variable for years is unnecessary. The primary differences between this equation and M. Hanlon (2005) are the substitution of specific BTDs in place of their taxable income estimates and the emphasis on IFRS, not the US GAAP.

Sample selection. BTDs are not machine readable, so data must be hand collected from the footnote disclosures in the annual reports of the FTSE 100 for the years 2004 through 2010. Only the footnote disclosures under IFRS are included, which is the reason for the start date. With these parameters present, 600 companyyear observations are included. The prices utilized are as of each company's respective annual report date.

Some overly cautious individuals could question not having larger sample sizes in researching the influence of DTAs and DTLs on stock prices. However, this sample size is in fact larger than most research in this area. B. Lev and D. Nissim (2004) rely on 75 companies of the Fortune 500 as an example of sample size. Thus, the sample size of 100 companies is considered robust in this area, especially considering the labor-intensive nature of hand collecting data from footnote disclosures and therein significant cost.

Description of book-tax difference variables. The temporary differences reviewed in this research are gathered from hand collecting and then sorting information from financial statement footnote disclosures. International Accounting Standard 12 (IAS 12) requires these components to be disclosed.

The DTAs include the following categories: DEPR (property, plant, and equipment items), EB (employee benefits but not including stock or option compensation), ESOP (employee stock or option compensation), DEBT (allowances for doubtful accounts), INV (inventory adjustments), UNEARN (unearned income representing deferred revenues), DERIV (derivatives), INVEST (mark-to-market investments adjustments), EQUITY (share of subsidiary or overseas income), INTAN (intangible asset temporary differences), ITEMS (tax issues different than book for items other than tax losses), LOSSES (loss carryforwards, etc.), RESERV (restructuring and other contingency provisions), ACCRUE (accrued expenses), and OTHER 
(items not significant enough to be reported in any respective category save this catchall). The DTLs include the following categories: DEPR (property, plant, and equipment items), EB (employee benefits but not including stock or option compensation), ESOP (employee stock or option compensation), RECEIV (allowances for doubtful accounts), INV (inventory adjustments), DEFER (deferred revenues),

\section{Sample means for DTAs and DTLs, $£$ millions}

Table 6

\begin{tabular}{|c|c|c|c|c|c|c|c|}
\hline Variable & Mean & 2004 & 2005 & 2006 & 2007 & 2008 & 2009 \\
\hline \multicolumn{8}{|l|}{ DTAs } \\
\hline DEPR & 68.2 & 55.9 & 67.9 & 70.8 & 80.9 & 71.3 & 58.6 \\
\hline EB & 272.7 & 326.8 & 329.2 & 248.1 & 196.6 & 240.4 & 294.9 \\
\hline ESOP & 38.7 & 17.4 & 34.0 & 56.8 & 48.6 & 36.8 & 38.5 \\
\hline DEBT & 925.2 & 562.0 & 528.2 & 748.0 & $1,175.9$ & $1,322.1$ & $1,215.0$ \\
\hline INV & 214.8 & 302.2 & 298.1 & 225.5 & 168.1 & 145.5 & 149.6 \\
\hline UNEARN & 201.7 & 393.4 & 322.0 & 160.0 & 153.9 & 104.6 & 76.1 \\
\hline DERIV & 100.9 & 11.9 & 104.3 & 95.2 & 100.8 & 215.0 & 78.3 \\
\hline INVEST & 191.7 & 71.8 & 142.1 & 132.0 & 125.1 & 445.2 & 233.9 \\
\hline EQUITY & 833.3 & 759.0 & 363.6 & 934.0 & $1,140.0$ & 620.0 & $1,183.0$ \\
\hline INTAN & 44.7 & 72.2 & 62.7 & 45.2 & 55.5 & 14.3 & 18.4 \\
\hline ITEMS & 192.7 & 89.9 & 171.6 & 10.4 & 37.0 & 283.0 & 564.0 \\
\hline LOSSES & 218.8 & 156.6 & 111.6 & 133.4 & 203.0 & 298.7 & 409.5 \\
\hline RESERV & 364.2 & 301.8 & 292.9 & 322.1 & 405.2 & 383.5 & 479.7 \\
\hline ACCRUE & 153.4 & 179.4 & 95.6 & 139.2 & 144.2 & 164.8 & 197.5 \\
\hline OTHER & 183.4 & 170.8 & 158.9 & 176.9 & 202.9 & 176.5 & 214.6 \\
\hline \multicolumn{8}{|l|}{ DTLs } \\
\hline DEPR & $1,116.0$ & $1,020.2$ & 969.2 & $1,015.7$ & $1,149.7$ & $1,224.7$ & $1,316.3$ \\
\hline EB & 153.6 & 173.8 & 152.9 & 150.5 & 176.8 & 126.6 & 140.9 \\
\hline ESOP & 51.2 & 22.0 & 121.0 & 56.3 & 37.0 & 12.8 & 58.0 \\
\hline RECEIV & 77.8 & 170.0 & 180.2 & 44.0 & 2.0 & 20.3 & 50.3 \\
\hline INV & 51.9 & 60.3 & 45.3 & 42.4 & 48.0 & 66.2 & 49.2 \\
\hline DEFER & 507.7 & 393.9 & 347.9 & 570.0 & 433.9 & 697.8 & 602.5 \\
\hline DERIV & 84.4 & 73.7 & 72.3 & 63.5 & 91.7 & 120.4 & 84.6 \\
\hline $\mathrm{FV}$ & 425.5 & 633.3 & 468.3 & 451.6 & 430.2 & 270.7 & 299.0 \\
\hline EQUITY & 333.7 & 316.5 & 355.7 & 242.2 & 292.4 & 346.7 & 448.8 \\
\hline INTAN & 343.2 & 203.9 & 249.6 & 264.8 & 434.1 & 454.3 & 452.5 \\
\hline LOSSES & 116.1 & 77.9 & 55.4 & 46.7 & 73.6 & 156.4 & 286.9 \\
\hline CONV & 5.1 & 6.2 & 8.1 & 4.5 & 2.2 & 3.9 & 6.0 \\
\hline PROV & 479.4 & 247.8 & 330.3 & 375.9 & 603.1 & 511.4 & 808.1 \\
\hline ACCRUE & 70.0 & 10.6 & 5.9 & 66.0 & 38.2 & 174.0 & 125.4 \\
\hline OTHER & 206.0 & 177.8 & 208.1 & 209.9 & 267.9 & 172.6 & 199.6 \\
\hline
\end{tabular}

The averages for each year over the six years of data for DTAs in millions are the following: £68.2404 DEPR, £272.6766 EB, £38.6793 ESOP, £925.2146 DEBT, £214.8204 INV, £201.6497 UNEARN, £100.9102 DERIV, £191.7001 INVEST, £833.2667 EQUITY, £44.7308 INTAN, £192.6608 ITEMS, £218.8086 LOSSES, £364.1928 RESERV, £153.4376 ACCRUE, and $£ 183.4191$ OTHER. The averages for each year over the six years of data for DTLs in millions are the following: $£ 1,115.95$ DEPR, £153.57 EB, £51.18 ESOP, £77.81 RECEIV, £51.90 INV, £507.65 DEFER, £84.36 DERIV, £425.52 FV, £333.72 EQUITY, $£ 343.20$ INTAN, £116.14 LOSSES, £5.125 CONV, £479.44 PROV, £70.02 ACCRUE, and £205.97 0THER. 
DERIV (derivatives), EQUITY (share of subsidiary or overseas income), INTAN (intangible asset temporary differences), LOSSES (loss carryforwards, etc.), CONV (convertible debt), PROV (restructuring and other contingency reserves), ACCRUE (accrued expenses), and OTHER (items not significant enough to be reported in any respective category save this catchall). To create the regression variables that measure surprise, the previous year's balance in that DTAs or DTLs category is subtracted from the current year's balance in that DTAs or DTLs category.

Table 6 discloses the averages within each category over each year. The DTLs DEPR has the largest average ( $£ 1,116.0$ million). The next largest DTLs is DEFER

Sample means for changes in DTAs and DTLs, $£$ millions

\begin{tabular}{|c|c|c|c|c|c|c|}
\hline Variable & Mean & 2004-05 & 2005-06 & 2006-07 & 2007-08 & 2008-09 \\
\hline \multicolumn{7}{|l|}{ DTAs } \\
\hline DEPR & .4 & 2.2 & 1.5 & 4.5 & (3.0) & $(3.2)$ \\
\hline EB & 6.4 & 17.6 & $(32.3)$ & \begin{tabular}{|l|}
$(40.6)$ \\
\end{tabular} & 43.5 & 44.0 \\
\hline ESOP & 2.1 & 3.0 & 10.7 & $(.4)$ & $(3.9)$ & 1.4 \\
\hline DEBT & 10.1 & $(1.3)$ & 8.8 & 17.1 & 19.1 & 6.8 \\
\hline INV & .3 & $(.1)$ & .1 & 1.1 & .1 & .3 \\
\hline UNEARN & $(2.3)$ & (3.6) & (3.3) & $(.5)$ & $(.8)$ & $(3.1)$ \\
\hline DERIV & 2.2 & 11.9 & 3.7 & 1.0 & 13.0 & (18.4) \\
\hline INVEST & 8.2 & 11.3 & $(1.2)$ & 4.2 & 91.3 & $(64.5)$ \\
\hline EQUITY & .8 & $(.3)$ & 2.1 & 2.1 & 1.0 & $(.6)$ \\
\hline INTAN & $(1.0)$ & .4 & (3.3) & .3 & $(2.9)$ & .3 \\
\hline ITEMS & 1.9 & 1.6 & (3.2) & .5 & 4.9 & 5.6 \\
\hline LOSSES & 30.8 & $(17.2)$ & 8.7 & 39.0 & 58.7 & 65.1 \\
\hline RESERV & 18.1 & 3.7 & 23.7 & 21.7 & 8.8 & 32.7 \\
\hline ACCRUE & 1.3 & $(2.4)$ & .8 & 3.1 & 2.9 & 2.3 \\
\hline OTHER & 7.1 & .5 & 13.8 & 11.6 & $(22.5)$ & 32.2 \\
\hline \multicolumn{7}{|l|}{ DTLs } \\
\hline DEPR & 42.6 & $(36.7)$ & 94.4 & 81.5 & $(4.2)$ & 78.2 \\
\hline EB & $(1.4)$ & $(.5)$ & 2.6 & 21.4 & (23.9) & $(6.6)$ \\
\hline ESOP & .3 & 3.2 & (1.9) & $(.6)$ & $(.6)$ & 1.2 \\
\hline RECEIV & $(.4)$ & .2 & $(3.2)$ & $(.4)$ & .2 & 1.3 \\
\hline INV & .3 & .6 & $(.1)$ & .2 & 1.4 & $(.9)$ \\
\hline DEFER & 9.4 & 3.3 & 16.5 & $(3.6)$ & 36.0 & $(5.4)$ \\
\hline DERIV & 1.4 & 2.9 & .0 & 5.0 & \begin{tabular}{|l|}
6.8 \\
\end{tabular} & $(7.5)$ \\
\hline $\mathrm{FV}$ & $(8.7)$ & $(3.5)$ & $(9.3)$ & $(6.0)$ & $(52.8)$ & 28.0 \\
\hline EQUITY & 4.1 & 8.3 & $(2.6)$ & 3.2 & \begin{tabular}{|l|}
8.8 \\
\end{tabular} & 2.9 \\
\hline INTAN & \begin{tabular}{|l|}
25.7 \\
\end{tabular} & 20.3 & 24.5 & 63.8 & 24.9 & $(5.2)$ \\
\hline LOSSES & 2.9 & .6 & $(1.9)$ & 3.2 & 5.1 & 7.6 \\
\hline $\mathrm{CONV}$ & .0 & .1 & $(.1)$ & .0 & .0 & .0 \\
\hline PROV & 10.9 & 8.3 & 13.4 & 28.1 & $(12.8)$ & 17.5 \\
\hline ACCRUE & 1.5 & $(.1)$ & 1.2 & .2 & 5.4 & .6 \\
\hline OTHER & 5.5 & 16.0 & 9.4 & 35.7 & $(46.7)$ & 13.0 \\
\hline
\end{tabular}




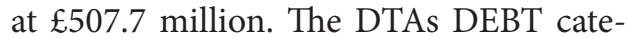
gory increases the most over time, which is representative of the financial situation worldwide ( $£ 528.2$ million low in 2005 to $£ 1,322.1$ million high in 2008).

Table 7 discusses the average change in each category over each year. The DTLs DEPR has the largest average change from one year to the next at $£ 42.6$ million. The next largest average change from year to year for DTLs is the category of INTAN at $£ 25.7$ million. The DTAs LOSSES has the largest change from year to year at $£ 30.8$ million. The next largest change in DTAs is the category of RESERV at $£ 18.1$ million.

\section{Results}

Table 8 shows the coefficient estimates of the DTAs and DTLs. Under the efficient market condition, the following are statistically significant: DTAs of DEBT and DTLs of DEPR, FV, INTAN and PROV. Under

Regression of changes in price on changes in aggregate DTA and DTL

Table 8

\begin{tabular}{|c|c|c|c|c|c|c|c|}
\hline DTAs Variable & 04-09 & $05-10$ & 04-10 & DTLs Variable & 04-09 & 05-10 & 04-10 \\
\hline DEPR & $\begin{array}{l}.202 \\
(.825)\end{array}$ & $\begin{array}{l}.155 \\
(.618)\end{array}$ & $\begin{array}{l}-.075 \\
(-.311)\end{array}$ & DEPR & $\begin{array}{l}.529^{\star} \\
(1.929)\end{array}$ & $\begin{array}{l}.188 \\
(.667)\end{array}$ & $\begin{array}{l}-.790^{\star \star} \\
(-2.911)\end{array}$ \\
\hline EB & $\begin{array}{l}.119 \\
(-.577)\end{array}$ & $\begin{array}{l}-.117 \\
(-.551)\end{array}$ & $\begin{array}{l}.024 \\
(-.117)\end{array}$ & EB & \begin{tabular}{|l|}
-2.249 \\
$(-1.864)$
\end{tabular} & $\begin{array}{l}-1.659 \\
(-1.338)\end{array}$ & $\begin{array}{l}1.033 \\
(.865)\end{array}$ \\
\hline ESOP & $\begin{array}{l}-.101 \\
(-.449)\end{array}$ & $\begin{array}{l}.148 \\
(-.640)\end{array}$ & $\begin{array}{l}.152 \\
(-.682)\end{array}$ & ESOP & $\begin{array}{l}.130 \\
(1.081)\end{array}$ & $\begin{array}{l}.080 \\
(.644)\end{array}$ & $\begin{array}{l}.104 \\
(-.873)\end{array}$ \\
\hline DEBT & 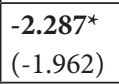 & \begin{tabular}{|l|}
-1.529 \\
$(-1.277)$ \\
\end{tabular} & $\begin{array}{l}1.476 \\
(1.280)\end{array}$ & RECEIV & \begin{tabular}{|l|}
-.575 \\
$(-1.548)$
\end{tabular} & $\begin{array}{l}.254 \\
(-.665)\end{array}$ & \begin{tabular}{|l|}
$.724^{\star}$ \\
$(1.970)$
\end{tabular} \\
\hline INV & $\begin{array}{l}.123 \\
(.727)\end{array}$ & $\begin{array}{l}114 \\
(.660) \\
\end{array}$ & $\begin{array}{l}.008 \\
(.051) \\
\end{array}$ & INV & \begin{tabular}{|l|}
.120 \\
$(.475)$
\end{tabular} & $\begin{array}{l}.159 \\
(.612)\end{array}$ & \begin{tabular}{|l|}
.135 \\
$(.541)$
\end{tabular} \\
\hline UNEARN & $\begin{array}{l}.012 \\
(.030) \\
\end{array}$ & \begin{tabular}{|l|}
-.403 \\
$(-.936)$ \\
\end{tabular} & $\begin{array}{l}-1.120^{* *} \\
(-2.700)\end{array}$ & DEFER & \begin{tabular}{|l|}
-.552 \\
$(-1.092)$ \\
\end{tabular} & $\begin{array}{l}.546 \\
(-1.052) \\
\end{array}$ & \begin{tabular}{|l|}
-.123 \\
$(-.246)$ \\
\end{tabular} \\
\hline DERIV & $\begin{array}{l}.112 \\
(-.505) \\
\end{array}$ & \begin{tabular}{|l|}
-.016 \\
$(-.071)$ \\
\end{tabular} & $\begin{array}{l}.232 \\
(1.053) \\
\end{array}$ & DERIV & \begin{tabular}{|l|}
.524 \\
$(1.544)$ \\
\end{tabular} & $\begin{array}{l}.513 \\
(1.469) \\
\end{array}$ & \begin{tabular}{|l|}
.100 \\
$(.297)$ \\
\end{tabular} \\
\hline INVEST & $\begin{array}{l}.327 \\
(-1.015)\end{array}$ & \begin{tabular}{|l|}
-.250 \\
$(-.756)$
\end{tabular} & $\begin{array}{l}.126 \\
.395)\end{array}$ & FV & \begin{tabular}{|l|}
$.490^{\star}$ \\
$(2.317)$
\end{tabular} & $\begin{array}{l}. \mathbf{6 0 8}^{\star \star} \\
(2.794)\end{array}$ & \begin{tabular}{|l|}
$.440^{\star}$ \\
$(2.100)$
\end{tabular} \\
\hline EQUITY & $\begin{array}{l}.195 \\
(-.901)\end{array}$ & $\begin{array}{l}-270 \\
(-1.212)\end{array}$ & $\begin{array}{l}.251 \\
(-1.170)\end{array}$ & EQUITY & \begin{tabular}{|l|}
.063 \\
$(.349)$
\end{tabular} & $\begin{array}{l}.012 \\
(-.065)\end{array}$ & \begin{tabular}{|l|}
-186 \\
$(-1.049)$
\end{tabular} \\
\hline INTAN & $\begin{array}{l}.259 \\
(1.337)\end{array}$ & $\begin{array}{l}.270 \\
(1.356)\end{array}$ & $\begin{array}{l}.094 \\
(.492) \\
\end{array}$ & INTAN & \begin{tabular}{|l|}
$\mathbf{5 5 0} \mathbf{5}^{\star}$ \\
$(2.222)$
\end{tabular} & $\begin{array}{l}.521^{\star} \\
(2.048)\end{array}$ & \begin{tabular}{|l|}
.059 \\
$(.241)$ \\
\end{tabular} \\
\hline ITEMS & $\begin{array}{l}.135 \\
(.328)\end{array}$ & $\begin{array}{l}.222 \\
(-.525)\end{array}$ & $\begin{array}{l}.932^{\star} \\
(-2.287)\end{array}$ & LOSSES & \begin{tabular}{|l|}
-.048 \\
$(-.389)$ \\
\end{tabular} & $\begin{array}{l}.025 \\
(.198)\end{array}$ & \begin{tabular}{|l|}
.185 \\
$(1.522)$
\end{tabular} \\
\hline LOSSES & $\begin{array}{l}.113 \\
(-.411) \\
\end{array}$ & \begin{tabular}{|l|}
-.215 \\
$(-.760)$ \\
\end{tabular} & $\begin{array}{l}.304 \\
(-1.114) \\
\end{array}$ & CONV & \begin{tabular}{|l|}
-.073 \\
$(-.637)$ \\
\end{tabular} & $\begin{array}{l}.052 \\
(-.436) \\
\end{array}$ & \begin{tabular}{|l|}
.040 \\
$(.355)$ \\
\end{tabular} \\
\hline RESERV & $\begin{array}{l}.061 \\
(.158) \\
\end{array}$ & \begin{tabular}{|l|}
.288 \\
$(.728)$ \\
\end{tabular} & $\begin{array}{l}.629 \\
(1.653) \\
\end{array}$ & PROV & \begin{tabular}{|l|}
$\mathbf{8 8 4}^{\star}$ \\
$(2.147)$ \\
\end{tabular} & \begin{tabular}{|l|}
.567 \\
$(1.339)$ \\
\end{tabular} & \begin{tabular}{|l|}
-.636 \\
$(-1.562)$ \\
\end{tabular} \\
\hline ACCRUE & $\begin{array}{l}.172 \\
(-.542)\end{array}$ & \begin{tabular}{|l|}
-.304 \\
$(-.931)$
\end{tabular} & $\begin{array}{l}.399 \\
(-1.268) \\
\end{array}$ & ACCRUE & \begin{tabular}{|l|}
.016 \\
$(.061)$ \\
\end{tabular} & $\begin{array}{l}.043 \\
(-.164)\end{array}$ & \begin{tabular}{|l|}
-.155 \\
$(-.611)$
\end{tabular} \\
\hline OTHER & $\begin{array}{l}.354 \\
(-1.117)\end{array}$ & \begin{tabular}{|l|}
-.205 \\
$(-.628)$
\end{tabular} & $\begin{array}{l}315 \\
(1.005)\end{array}$ & OTHER & \begin{tabular}{|l|}
-.123 \\
$(-.431)$
\end{tabular} & $\begin{array}{l}.026 \\
(-.090)\end{array}$ & \begin{tabular}{|l|}
.231 \\
$(.814)$ \\
\end{tabular} \\
\hline
\end{tabular}


the less than efficient market condition, the following are statistically significant: DTLs of FV and INTAN. If the entire horizon of prices is considered, the DTAs of UNEARN and ITEMS and the DTLs of DEPR, RECEIV, and FV are statistically significant. If all these items are considered together, the DTLs of DEPR, FV and INTAN would seem to be the most powerful. They exist under two of the proposed market conditions. Potentially, the DTA of UNEARN could be considered in that category because of its powerful statistical significance.

The components underlying the DTLs of DEPR and INTAN book-tax differences are not disclosed anywhere else in the financial statements. Thus, these two categories could be the reason for the strength of DTLs as an influence on stock prices. The components of FV are disclosed elsewhere in the financial statements but not in so collected the number as FV here.

Regression involving changes in price and DTAs and DTLs, 2004 through 2009

Table 9

\begin{tabular}{|c|c|c|c|c|c|c|c|c|c|c|c|}
\hline $\begin{array}{l}\text { DTAs } \\
\text { Variable }\end{array}$ & 04-05 & 05-06 & 06-07 & 07-08 & 08-09 & $\begin{array}{l}\text { DTLs } \\
\text { Variable }\end{array}$ & 04-05 & 05-06 & $06-07$ & 07-08 & 08-09 \\
\hline DEPR & $\begin{array}{l}.161 \\
(1.132) \\
\end{array}$ & \begin{tabular}{|l|}
268 \\
$(1.106)$
\end{tabular} & \begin{tabular}{|l}
-.005 \\
$(-.015)$
\end{tabular} & \begin{tabular}{|l}
-.143 \\
$(-.961)$
\end{tabular} & \begin{tabular}{|l|}
.237 \\
$(-.871)$ \\
\end{tabular} & DEPR & \begin{tabular}{|l|}
$.490^{\star}$ \\
$(-2.052)$ \\
\end{tabular} & \begin{tabular}{|l}
.163 \\
$(.549)$ \\
\end{tabular} & $\begin{array}{l}\mathbf{7 5 1} 1^{\star *} \\
(3.489)\end{array}$ & \begin{tabular}{|l|}
.027 \\
$(.239)$ \\
\end{tabular} & \begin{tabular}{|l}
.291 \\
$(1.302)$ \\
\end{tabular} \\
\hline EB & \begin{tabular}{|l|}
.071 \\
$.383)$
\end{tabular} & $\begin{array}{l}163 \\
(.718) \\
\end{array}$ & \begin{tabular}{|l|}
-.100 \\
$(-.517)$
\end{tabular} & \begin{tabular}{|l|}
-.262 \\
$(-1.484)$ \\
\end{tabular} & \begin{tabular}{|l|}
-185 \\
$(-1.255)$ \\
\end{tabular} & EB & \begin{tabular}{|l|}
$1.365^{\star}$ \\
$(2.222)$
\end{tabular} & $\begin{array}{l}854 \\
(1.146) \\
\end{array}$ & $\begin{array}{l}.017 \\
(.134)\end{array}$ & \begin{tabular}{|l|}
-.455 \\
$(-1.285)$ \\
\end{tabular} & \begin{tabular}{|l}
.096 \\
$(.593)$ \\
\end{tabular} \\
\hline ESOP & \begin{tabular}{|l|}
$.337^{\star}$ \\
$(-2.061)$ \\
\end{tabular} & \begin{tabular}{|l|}
.160 \\
$(.337)$ \\
\end{tabular} & \begin{tabular}{|l|}
.058 \\
$(.301)$ \\
\end{tabular} & \begin{tabular}{|l|}
-.065 \\
$(-.430)$ \\
\end{tabular} & \begin{tabular}{|l|}
209 \\
$(1.534)$ \\
\end{tabular} & ESOP & \begin{tabular}{|l|}
.038 \\
$(-.247)$ \\
\end{tabular} & \begin{tabular}{|l|}
.118 \\
$(.310)$ \\
\end{tabular} & \begin{tabular}{|l|}
-120 \\
$(-1.434)$ \\
\end{tabular} & \begin{tabular}{|l|}
.082 \\
$(.976)$ \\
\end{tabular} & \begin{tabular}{|l|}
.093 \\
$(.880)$ \\
\end{tabular} \\
\hline DEBT & \begin{tabular}{|l|}
-.115 \\
$(-.286)$ \\
\end{tabular} & \begin{tabular}{|l|}
-.259 \\
$(-.061)$ \\
\end{tabular} & $\begin{array}{l}.025 \\
(.046)\end{array}$ & \begin{tabular}{|l|}
-.184 \\
$(-.602)$ \\
\end{tabular} & $\begin{array}{l}.833 \\
(1.635) \\
\end{array}$ & RECEIV & \begin{tabular}{|l|}
.156 \\
$(.490)$ \\
\end{tabular} & \begin{tabular}{|l|}
-.182 \\
$(-.043)$ \\
\end{tabular} & \begin{tabular}{|l}
.093 \\
$(.291)$ \\
\end{tabular} & \begin{tabular}{|l}
.019 \\
$(-.235)$ \\
\end{tabular} & \begin{tabular}{|l}
.259 \\
$(1.357)$ \\
\end{tabular} \\
\hline INV & \begin{tabular}{|l|}
354 \\
$(1.099)$ \\
\end{tabular} & \begin{tabular}{|l}
.005 \\
$(.010)$ \\
\end{tabular} & \begin{tabular}{|l|}
202 \\
$(1.161)$ \\
\end{tabular} & \begin{tabular}{|l|}
.172 \\
$(1.925)$ \\
\end{tabular} & \begin{tabular}{|l|}
.035 \\
$(-.230)$ \\
\end{tabular} & INV & \begin{tabular}{|l|}
160 \\
$(1.554)$ \\
\end{tabular} & & & \begin{tabular}{|l|}
.007 \\
$(.010)$ \\
\end{tabular} & \begin{tabular}{|l|l|}
1.880 \\
$(1.444)$ \\
\end{tabular} \\
\hline UNEARN & \begin{tabular}{|l|}
.212 \\
$(.398)$
\end{tabular} & \begin{tabular}{|l|}
-.247 \\
$(-.562)$ \\
\end{tabular} & \begin{tabular}{|l|}
.204 \\
$(-1.125)$ \\
\end{tabular} & \begin{tabular}{|l}
.423 \\
$(.643)$
\end{tabular} & \begin{tabular}{|l|}
.003 \\
$(-.007)$
\end{tabular} & DEFER & & & & $\begin{array}{l}1.215^{\star} \\
(2.195)\end{array}$ & \begin{tabular}{|l}
.144 \\
$(.497)$ \\
\end{tabular} \\
\hline DERIV & \begin{tabular}{|l}
.034 \\
$(.058)$ \\
\end{tabular} & \begin{tabular}{|l|}
.133 \\
$(-.301)$ \\
\end{tabular} & \begin{tabular}{|l|}
.048 \\
$(.172)$ \\
\end{tabular} & \begin{tabular}{|l|}
-225 \\
$(-1.335)$ \\
\end{tabular} & \begin{tabular}{|l}
.146 \\
$(.765)$ \\
\end{tabular} & DERIV & \begin{tabular}{|l}
.179 \\
$(.838)$ \\
\end{tabular} & \begin{tabular}{|l|}
-.071 \\
$(-.340)$ \\
\end{tabular} & \begin{tabular}{|l|}
.013 \\
$(.037)$ \\
\end{tabular} & \begin{tabular}{|l|}
-.389 \\
$(-1.526)$ \\
\end{tabular} & \begin{tabular}{|l}
.481 \\
$(1.686)$ \\
\end{tabular} \\
\hline INVEST & \begin{tabular}{|l}
.497 \\
$(.595)$ \\
\end{tabular} & \begin{tabular}{|l|}
-.155 \\
$(-.336)$ \\
\end{tabular} & \begin{tabular}{|l}
.054 \\
$(.316)$ \\
\end{tabular} & $\begin{array}{l}-1.112^{\star} \\
(-2.586)\end{array}$ & \begin{tabular}{|l|}
-.180 \\
$(-.445)$ \\
\end{tabular} & FV & $\begin{array}{l}.040 \\
(.179)\end{array}$ & $\begin{array}{l}-.047 \\
(-.213)\end{array}$ & $\begin{array}{l}-.032 \\
(-.272)\end{array}$ & \begin{tabular}{|l|}
-.268 \\
$(-1.553)$ \\
\end{tabular} & \begin{tabular}{|l}
$-.764^{\star}$ \\
$(-2.076)$ \\
\end{tabular} \\
\hline EQUITY & \begin{tabular}{|l|}
-.276 \\
$(-1.764)$ \\
\end{tabular} & \begin{tabular}{|l|}
-.041 \\
$(-.149)$ \\
\end{tabular} & \begin{tabular}{|l|}
.041 \\
$(.152)$ \\
\end{tabular} & \begin{tabular}{|l|}
.051 \\
$(.071)$ \\
\end{tabular} & \begin{tabular}{|l|}
-1.742 \\
$(-1.307)$ \\
\end{tabular} & EQUITY & \begin{tabular}{|l|}
.067 \\
$(.245)$ \\
\end{tabular} & \begin{tabular}{|l|}
.164 \\
$(.793)$ \\
\end{tabular} & \begin{tabular}{|l|}
-.021 \\
$(-.054)$ \\
\end{tabular} & \begin{tabular}{|l|}
.047 \\
$(.459)$ \\
\end{tabular} & \begin{tabular}{|l}
$\mathbf{3 2 1}^{\star}$ \\
$(2.128)$ \\
\end{tabular} \\
\hline INTAN & \begin{tabular}{|l|}
.018 \\
$(.157)$ \\
\end{tabular} & \begin{tabular}{|l|}
.159 \\
$(.934)$ \\
\end{tabular} & $\begin{array}{l}.086 \\
(.737) \\
\end{array}$ & \begin{tabular}{|l|}
.060 \\
$(.514)$
\end{tabular} & \begin{tabular}{|l|}
044 \\
$(.318)$ \\
\end{tabular} & INTAN & \begin{tabular}{|l|}
-.113 \\
$(-.599)$
\end{tabular} & \begin{tabular}{|l|}
269 \\
$(1.839)$
\end{tabular} & $\begin{array}{l}.114 \\
(.373) \\
\end{array}$ & \begin{tabular}{|l|}
199 \\
$(1.726)$
\end{tabular} & $\begin{array}{l}.121 \\
(.566) \\
\end{array}$ \\
\hline ITEMS & \begin{tabular}{|l|l}
347 \\
$(1.185)$ \\
\end{tabular} & \begin{tabular}{|l}
-.157 \\
$(-.333)$ \\
\end{tabular} & $\begin{array}{l}-.080 \\
(-.900) \\
\end{array}$ & $\begin{array}{l}.010 \\
(.016)\end{array}$ & $\begin{array}{l}.072 \\
(.354) \\
\end{array}$ & LOSSES & \begin{tabular}{|l|}
-240 \\
$(-1.160)$ \\
\end{tabular} & \begin{tabular}{|l|l}
-.015 \\
$(-.089)$ \\
\end{tabular} & \begin{tabular}{|l|}
.150 \\
$(.340)$ \\
\end{tabular} & \begin{tabular}{|l|l}
-.052 \\
$(-.406)$ \\
\end{tabular} & \begin{tabular}{|l}
-.079 \\
$(-.682)$ \\
\end{tabular} \\
\hline LOSSES & \begin{tabular}{|l|}
.492 \\
$(-1.155)$ \\
\end{tabular} & \begin{tabular}{|l|}
-.604 \\
$(-1.067)$
\end{tabular} & \begin{tabular}{|l|}
-.265 \\
$(-.855)$
\end{tabular} & $\begin{array}{l}. \mathbf{2 3 6} 6^{\star} \\
(2.189)\end{array}$ & \begin{tabular}{|l}
510 \\
$(1.053)$
\end{tabular} & CONV & \begin{tabular}{|l|}
.016 \\
$(-.080)$
\end{tabular} & \begin{tabular}{|l|}
-.061 \\
$(-.328)$
\end{tabular} & \begin{tabular}{|l|}
.005 \\
$(.059)$ \\
\end{tabular} & \begin{tabular}{|l}
.018 \\
$(.218)$ \\
\end{tabular} & \begin{tabular}{|l|}
-.025 \\
$(-.169)$ \\
\end{tabular} \\
\hline RESERV & \begin{tabular}{|l|}
$.772^{\star \star}$ \\
$(3.303)$ \\
\end{tabular} & \begin{tabular}{|l|}
-.266 \\
$(-.539)$ \\
\end{tabular} & \begin{tabular}{|l|}
346 \\
$(1.201)$ \\
\end{tabular} & \begin{tabular}{|l}
$\mathbf{8 4 9}^{\star}$ \\
$(2.302)$ \\
\end{tabular} & \begin{tabular}{|l}
.138 \\
$(.504)$ \\
\end{tabular} & PROV & \begin{tabular}{|l|}
$-.937^{\star}$ \\
$(-2.580)$ \\
\end{tabular} & $\begin{array}{l}.002 \\
(.006) \\
\end{array}$ & \begin{tabular}{|l}
.264 \\
$(1.401)$ \\
\end{tabular} & \begin{tabular}{|l}
-118 \\
$(-.831)$ \\
\end{tabular} & \begin{tabular}{|l}
-.486 \\
$(-1.294)$ \\
\end{tabular} \\
\hline ACCRUE & \begin{tabular}{|l|}
-.206 \\
$(-.452)$
\end{tabular} & $\begin{array}{l}-.138 \\
(-.339) \\
\end{array}$ & \begin{tabular}{|l|}
-.422 \\
$(-1.045)$ \\
\end{tabular} & $\begin{array}{l}.191 \\
(.321) \\
\end{array}$ & \begin{tabular}{|l|}
-.032 \\
$(-.191)$
\end{tabular} & ACCRUE & \begin{tabular}{|l|}
-.013 \\
$(-.122)$
\end{tabular} & \begin{tabular}{|l|}
.008 \\
$(.055)$ \\
\end{tabular} & \begin{tabular}{|l|}
.033 \\
$(.330)$ \\
\end{tabular} & \begin{tabular}{|l|}
.204 \\
$(.620)$ \\
\end{tabular} & $\begin{array}{l}186 \\
(1.059)\end{array}$ \\
\hline OTHER & \begin{tabular}{|l|}
322 \\
$(1.244)$ \\
\end{tabular} & \begin{tabular}{|l}
.010 \\
$(.020)$ \\
\end{tabular} & \begin{tabular}{|l|}
.040 \\
$(.264)$ \\
\end{tabular} & $\begin{array}{l}-.069 \\
(-.250) \\
\end{array}$ & \begin{tabular}{|l|}
-.120 \\
$(-.382)$ \\
\end{tabular} & OTHER & $\begin{array}{l}-1.042^{\star} \\
(-2.161) \\
\end{array}$ & \begin{tabular}{|l|}
-.166 \\
$(-.658)$ \\
\end{tabular} & \begin{tabular}{|l|}
-.351 \\
$(-.671)$ \\
\end{tabular} & \begin{tabular}{|l|}
608 \\
$(1.635)$ \\
\end{tabular} & \begin{tabular}{|l|}
-347 \\
$(-1.423)$ \\
\end{tabular} \\
\hline
\end{tabular}


DEPR could show that companies are purchasing more capital equipment, which accentuates the differences between book and tax. Purchasing more capital equipment signals to the market an expectation of higher returns in future years. Officers would not invest resources in long-lived asset categories without expecting sufficient increases in earnings over future years to continue current returns on investments. This signal could be why DEPR is so relevant to stock price changes.

INTAN could demonstrate an identical expectation of future profitability margin increases but with regard to the companies' copyrights, patents, trademarks, and other related assets. This INTAN area then would be relevant to investors as well.

UNEARN could show the quality of accruals. This UNEARN category involves the deferral of revenues where the revenue must be recognized for tax but not yet for book.

Table 9 separates the previously discussed results into each year over the time under consideration but under the efficient market condition. From 2004 through 2005, the DTAs of ESOP and RESERV and the DTLs of DEPR, EB, PROV, and OTHER are statistically significant. From 2005 through 2006, no category is statistically significant. From 2006 through 2007, the DTL of DEPR is statistically significant. From 2007 through 2008, the DTAs of INVEST, LOSSES, and RESERV and the DTL of DEFER are statistically significant. From 2008 through 2009, the DTLs of FV and EQUITY are statistically significant. This result would seem to discourage the overall statistical significance of any category save for the DTL of DEPR as no other category is statistically significant even twice.

Table 10 continues the separation of the previously mentioned results into the year to year area but under the less than efficient market condition. From 2005 through 2006, no category is statistically significant. From 2006 through 2007, the DTAs of UNEARN, ITEMS, and RESERV and the DTLs of EB, DEFER, FV, and CONV are statistically significant. From 2007 through 2008, the DTA of LOSSES and DTLs of DEPR, FV, and INTAN are statistically significant. From 2008 through 2009 , no category is statistically significant. From 2009 through 2010, the previous time period's answer continues. This result would also seem to discourage the overall statistical significance of any category this time save for the DTL of FV as no other category is statistically significant even twice.

Then, discussion turns to whether investors' stock price reactions are based on surprises. The changes from 2005 through 2006 in DTAs and DTLs are compared to the changes from 2004 through 2005 in DTAs and DTLs. This comparison continues until all the DTAs and DTLs for the time period are considered. As the reason for this procedure, investors could anticipate changes in DTAs and DTLs to continue at the identical number from year to year. Thus, any number for the change different than expected from year to year would prompt some stock price reaction from investors.

Table 11 involves consideration of year to year measures of surprises under the efficient market condition. From 2005 through 2006, no category is statistically significant. From 2006 through 2007, the DTAs of DERIV and EQUITY and the DTLs of INV and FV are statistically 
Regression involving changes in price and DTAs and DTLs, 2005 through 2010

Table 10

\begin{tabular}{|c|c|c|c|c|c|c|c|c|c|c|c|}
\hline $\begin{array}{l}\text { DTAs } \\
\text { Variable }\end{array}$ & 05-06 & 06-07 & 07-08 & 08-09 & 09-10 & $\begin{array}{l}\text { DTLs } \\
\text { Variable }\end{array}$ & 05-06 & 06-07 & 07-08 & 08-09 & 09-10 \\
\hline DEPR & \begin{tabular}{|l|}
-.023 \\
$(-.134)$ \\
\end{tabular} & $\begin{array}{l}.160 \\
(-.864) \\
\end{array}$ & \begin{tabular}{|l}
319 \\
$(1.063)$ \\
\end{tabular} & \begin{tabular}{|l|}
-.087 \\
$(-.435)$ \\
\end{tabular} & \begin{tabular}{|l}
.306 \\
$(-1.035)$ \\
\end{tabular} & DEPR & \begin{tabular}{|l|}
-.059 \\
$(-.206)$ \\
\end{tabular} & \begin{tabular}{|l|}
.123 \\
$(-.544)$ \\
\end{tabular} & \begin{tabular}{|l|}
$.685^{\star *}$ \\
$(-3.924)$ \\
\end{tabular} & \begin{tabular}{|l|}
-.053 \\
$(-.344)$ \\
\end{tabular} & \begin{tabular}{|l|}
-308 \\
$(-1.271)$ \\
\end{tabular} \\
\hline EB & \begin{tabular}{|l|}
.031 \\
$(.141)$
\end{tabular} & \begin{tabular}{|l|}
.081 \\
$(.465)$
\end{tabular} & \begin{tabular}{|l|}
.115 \\
$.734)$
\end{tabular} & $\begin{array}{l}.199 \\
(.838)\end{array}$ & \begin{tabular}{|l|}
.008 \\
$(.047)$ \\
\end{tabular} & EB & \begin{tabular}{|l|}
-.676 \\
$(-.924)$ \\
\end{tabular} & $\begin{array}{l}-1.291^{\star} \\
(-2.267)\end{array}$ & \begin{tabular}{|l|}
.000 \\
$(-.003)$
\end{tabular} & \begin{tabular}{|l|}
.375 \\
$(.788)$
\end{tabular} & \begin{tabular}{|l|}
-.023 \\
$(-.132)$
\end{tabular} \\
\hline ESOP & $\begin{array}{l}-.054 \\
(-.277) \\
\end{array}$ & \begin{tabular}{|l|}
-.029 \\
$(-.079)$ \\
\end{tabular} & \begin{tabular}{|l|}
-.018 \\
$(-.112)$
\end{tabular} & \begin{tabular}{|l|}
.177 \\
$(.868)$ \\
\end{tabular} & \begin{tabular}{|l|}
-.031 \\
$(-.210)$
\end{tabular} & ESOP & \begin{tabular}{|l|}
.085 \\
$(.465)$ \\
\end{tabular} & \begin{tabular}{|l|}
.111 \\
$(.384)$ \\
\end{tabular} & $\begin{array}{l}.135 \\
(1.987)\end{array}$ & \begin{tabular}{|l|}
-.072 \\
$(-.644)$
\end{tabular} & \begin{tabular}{|l|}
.069 \\
$(.601)$
\end{tabular} \\
\hline DEBT & \begin{tabular}{|l|}
-.208 \\
$(-.436)$
\end{tabular} & $\begin{array}{l}3.342 \\
(1.035)\end{array}$ & \begin{tabular}{|l|}
.291 \\
$(.663)$
\end{tabular} & \begin{tabular}{|l}
.097 \\
$(.236)$
\end{tabular} & \begin{tabular}{|l|}
.472 \\
$(.852)$
\end{tabular} & RECEIV & \begin{tabular}{|l|}
-.190 \\
$(-.501)$ \\
\end{tabular} & \begin{tabular}{|l|}
3.690 \\
$(1.152)$
\end{tabular} & $\begin{array}{l}393 \\
(1.510)\end{array}$ & $\begin{array}{l}.086 \\
(.771)\end{array}$ & $\begin{array}{l}.208 \\
(1.004)\end{array}$ \\
\hline INV & \begin{tabular}{|l}
.040 \\
$(.105)$ \\
\end{tabular} & \begin{tabular}{|l|}
-.052 \\
$(-.135)$ \\
\end{tabular} & \begin{tabular}{|l|}
-247 \\
$(-1.746)$ \\
\end{tabular} & $\begin{array}{l}-.099 \\
(-.824) \\
\end{array}$ & \begin{tabular}{|l|}
-.093 \\
$(-.560)$ \\
\end{tabular} & INV & \begin{tabular}{|l|}
-.029 \\
$(-.236)$ \\
\end{tabular} & \begin{tabular}{|l|}
-.024 \\
$(-.117)$ \\
\end{tabular} & \begin{tabular}{|l|}
-.450 \\
$(-1.130)$ \\
\end{tabular} & \begin{tabular}{|l|}
-.038 \\
$(-.039)$ \\
\end{tabular} & \begin{tabular}{|l|}
1.049 \\
$(.741)$ \\
\end{tabular} \\
\hline UNEARN & $\begin{array}{l}.383 \\
(.603)\end{array}$ & \begin{tabular}{|l|}
$.791^{\star}$ \\
$(2.362)$
\end{tabular} & $\begin{array}{l}-133 \\
(-.901)\end{array}$ & $\begin{array}{l}-.401 \\
(-.455)\end{array}$ & $\begin{array}{l}-.260 \\
(-.587)\end{array}$ & DEFER & \begin{tabular}{|l|}
.550 \\
$(.880)$ \\
\end{tabular} & $\begin{array}{l}.477^{\star} \\
(2.285)\end{array}$ & \begin{tabular}{|l|}
-172 \\
$(-1.261)$
\end{tabular} & \begin{tabular}{|l|}
-378 \\
$(-.509)$
\end{tabular} & $\begin{array}{l}.157 \\
(.496)\end{array}$ \\
\hline DERIV & $\begin{array}{l}.411 \\
(.578) \\
\end{array}$ & \begin{tabular}{|l|}
-.076 \\
$(-.225)$ \\
\end{tabular} & \begin{tabular}{|l|}
-.266 \\
$(-1.172)$ \\
\end{tabular} & $\begin{array}{l}.004 \\
(.017) \\
\end{array}$ & $\begin{array}{l}.072 \\
(.346) \\
\end{array}$ & DERIV & \begin{tabular}{|l|}
-.236 \\
$(-.927)$ \\
\end{tabular} & $\begin{array}{l}.136 \\
(.851) \\
\end{array}$ & \begin{tabular}{|l|}
.000 \\
$(.000)$
\end{tabular} & $\begin{array}{l}.181 \\
(.528) \\
\end{array}$ & $\begin{array}{l}.044 \\
(.143) \\
\end{array}$ \\
\hline INVEST & \begin{tabular}{|l|}
-.246 \\
$(-.247)$ \\
\end{tabular} & $\begin{array}{l}.024 \\
(.067) \\
\end{array}$ & \begin{tabular}{|l|}
-.055 \\
$(-.392)$ \\
\end{tabular} & \begin{tabular}{|l|}
.262 \\
$(.452)$ \\
\end{tabular} & $\begin{array}{l}.131 \\
(.297) \\
\end{array}$ & FV & \begin{tabular}{|l|}
299 \\
$(1.114)$ \\
\end{tabular} & \begin{tabular}{|l|}
$.575^{\star \star}$ \\
$(3.418)$ \\
\end{tabular} & \begin{tabular}{|l}
$.285^{\star \star}$ \\
$(-3.006)$ \\
\end{tabular} & \begin{tabular}{|l|}
.282 \\
$(1.215)$ \\
\end{tabular} & \begin{tabular}{|l|}
-.519 \\
$(-1.297)$ \\
\end{tabular} \\
\hline EQUITY & \begin{tabular}{|l|}
.083 \\
$(.452)$ \\
\end{tabular} & \begin{tabular}{|l|}
-.033 \\
$(-.158)$ \\
\end{tabular} & \begin{tabular}{|l|}
259 \\
$(1.179)$ \\
\end{tabular} & \begin{tabular}{|l|}
.025 \\
$(.026)$ \\
\end{tabular} & \begin{tabular}{|l|}
-.946 \\
$(-.653)$ \\
\end{tabular} & EQUITY & \begin{tabular}{|l|}
.122 \\
$(.376)$ \\
\end{tabular} & \begin{tabular}{|l|}
-.188 \\
$(-1.186)$ \\
\end{tabular} & \begin{tabular}{|l|}
-.125 \\
$(-.399)$ \\
\end{tabular} & \begin{tabular}{|l|}
.076 \\
$(.554)$ \\
\end{tabular} & \begin{tabular}{|l|}
.140 \\
$(.852)$ \\
\end{tabular} \\
\hline INTAN & \begin{tabular}{|l|}
-.233 \\
$(-1.676)$
\end{tabular} & \begin{tabular}{|l|}
-.029 \\
$(-.225)$ \\
\end{tabular} & $\begin{array}{l}.044 \\
(.469) \\
\end{array}$ & \begin{tabular}{|l|}
.031 \\
$(.194)$ \\
\end{tabular} & \begin{tabular}{|l|}
-.042 \\
$(-.279)$
\end{tabular} & INTAN & $\begin{array}{l}.039 \\
(.173) \\
\end{array}$ & \begin{tabular}{|l|}
-.131 \\
$(-1.175)$
\end{tabular} & $\begin{array}{l}.527^{\star} \\
(2.133)\end{array}$ & \begin{tabular}{|l|}
-.044 \\
$(-.283)$
\end{tabular} & \begin{tabular}{|l|}
-.019 \\
$(-.082)$
\end{tabular} \\
\hline ITEMS & \begin{tabular}{|l|}
.148 \\
$(.424)$ \\
\end{tabular} & \begin{tabular}{|l|}
$.755^{\star}$ \\
$(2.096)$
\end{tabular} & \begin{tabular}{|l|}
.022 \\
$(.309)$ \\
\end{tabular} & $\begin{array}{l}-.091 \\
(-.109) \\
\end{array}$ & \begin{tabular}{|l|}
.086 \\
$(.392)$ \\
\end{tabular} & LOSSES & \begin{tabular}{|l|}
.207 \\
$(.840)$ \\
\end{tabular} & $\begin{array}{l}.161 \\
(1.286) \\
\end{array}$ & $\begin{array}{l}.079 \\
(.220) \\
\end{array}$ & \begin{tabular}{|l|}
-.002 \\
$(-.013)$ \\
\end{tabular} & \begin{tabular}{|l|}
.063 \\
$(.495)$ \\
\end{tabular} \\
\hline LOSSES & \begin{tabular}{|l|}
382 \\
$(.754)$ \\
\end{tabular} & \begin{tabular}{|l|}
.526 \\
$(1.215)$ \\
\end{tabular} & $\begin{array}{l}.593^{*} \\
(-2.355) \\
\end{array}$ & \begin{tabular}{|l|}
-163 \\
$(-1.125)$ \\
\end{tabular} & \begin{tabular}{|l|}
-.120 \\
$(-.228)$ \\
\end{tabular} & CONV & \begin{tabular}{|l|}
.298 \\
$(1.282)$ \\
\end{tabular} & $\begin{array}{l}.395^{\star *} \\
(-2.772) \\
\end{array}$ & \begin{tabular}{|l|}
.041 \\
$(.611)$ \\
\end{tabular} & \begin{tabular}{|l|}
-.014 \\
$(-.122)$ \\
\end{tabular} & $\begin{array}{l}.030 \\
(.182) \\
\end{array}$ \\
\hline RESERV & \begin{tabular}{|l|}
-.170 \\
$(-.609)$ \\
\end{tabular} & $\begin{array}{l}1.358^{\star *} \\
(3.604)\end{array}$ & \begin{tabular}{|l|}
.430 \\
$(1.836)$ \\
\end{tabular} & \begin{tabular}{|l|}
-.768 \\
$(-1.551)$ \\
\end{tabular} & \begin{tabular}{|l|}
.080 \\
$(.267)$ \\
\end{tabular} & PROV & \begin{tabular}{|l|}
.015 \\
$(.035)$ \\
\end{tabular} & \begin{tabular}{|l|}
-.439 \\
$(-1.368)$ \\
\end{tabular} & \begin{tabular}{|l}
.083 \\
$(.542)$ \\
\end{tabular} & \begin{tabular}{|l|}
.089 \\
$(.467)$ \\
\end{tabular} & \begin{tabular}{|l|}
271 \\
$(.662)$ \\
\end{tabular} \\
\hline ACCRUE & \begin{tabular}{|l|}
375 \\
$(.691)$ \\
\end{tabular} & $\begin{array}{l}.392 \\
(-1.260) \\
\end{array}$ & \begin{tabular}{|l|}
337 \\
$(1.028)$ \\
\end{tabular} & \begin{tabular}{|l|}
-.337 \\
$(-.421)$ \\
\end{tabular} & \begin{tabular}{|l|}
-.087 \\
$(-.479)$ \\
\end{tabular} & ACCRUE & \begin{tabular}{|l|}
.072 \\
$(.567)$ \\
\end{tabular} & \begin{tabular}{|l|}
-202 \\
$(-1.868)$ \\
\end{tabular} & \begin{tabular}{|l|}
-.041 \\
$(-.501)$ \\
\end{tabular} & \begin{tabular}{|l|}
-.045 \\
$(-.103)$ \\
\end{tabular} & \begin{tabular}{|l|}
.080 \\
$(.419)$ \\
\end{tabular} \\
\hline OTHER & \begin{tabular}{|l|}
.161 \\
$(.523)$ \\
\end{tabular} & \begin{tabular}{|l|}
.012 \\
$(.031)$ \\
\end{tabular} & \begin{tabular}{|l|}
-.035 \\
$(-.288)$ \\
\end{tabular} & \begin{tabular}{|l|}
-.217 \\
$(-.585)$ \\
\end{tabular} & \begin{tabular}{|l|}
-.257 \\
$(-.755)$ \\
\end{tabular} & OTHER & \begin{tabular}{|l|}
302 \\
$(.527)$
\end{tabular} & \begin{tabular}{|l|}
-346 \\
$(-1.796)$
\end{tabular} & \begin{tabular}{|l|}
-.123 \\
$(-.290)$
\end{tabular} & $\begin{array}{l}.352 \\
(-.706)\end{array}$ & $\begin{array}{l}.000 \\
(.001) \\
\end{array}$ \\
\hline
\end{tabular}

significant. From 2007 through 2008, the DTAs of DEBT, INV, LOSSES, RESERV, and ACCRUE and the DTL of DERIV are statistically significant. From 2008 through 2009, the DTA of RESERV is statistically significant. This result seems to counter the overall statistical significance of any category save for the DTA of RESERV as no other category is statistically significant even twice.

Table 12 deals with year to year measures of surprises under the less than efficient market condition. From 2006 through 2007, the DTAs of UNEARN, DERIV, and RESERV and the DTLs of DEFER and FV are statistically significant. From 2007 through 2008, the DTAs of DEBT, INV, DERIV, EQUITY, and LOSSES and the DTLs of DEPR, ESOP, RECEIV, INV, DERIV, FV, and INTAN are statistically significant. From 2008 through 2009, no category is statistically significant. From 
Table 11

Measuring surprise: regressing changes in price from 2005 through 2006 on differences between changes in 2005-06 DTAs and DTLs and changes in 2004-05 DTAs and DTLs

\begin{tabular}{|c|c|c|c|c|c|c|c|c|c|}
\hline $\begin{array}{l}\text { DTAs } \\
\text { Variable }\end{array}$ & 05-06 & $06-07$ & 07-08 & 08-09 & $\begin{array}{l}\text { DTLs } \\
\text { Variable }\end{array}$ & 05-06 & $06-07$ & 07-08 & 08-09 \\
\hline DEPR & $\begin{array}{l}.032 \\
(.153)\end{array}$ & \begin{tabular}{|l|}
-.124 \\
$(-.750)$
\end{tabular} & $\begin{array}{l}-.421 \\
(-1.508)\end{array}$ & $\begin{array}{l}.102 \\
(.513)\end{array}$ & DEPR & $\begin{array}{l}.151 \\
(.558)\end{array}$ & $\begin{array}{l}.465 \\
(1.826)\end{array}$ & $\begin{array}{l}.228 \\
(1.340)\end{array}$ & $\begin{array}{l}.110 \\
(.510)\end{array}$ \\
\hline EB & $\begin{array}{l}.178 \\
(.729)\end{array}$ & \begin{tabular}{|l|}
.134 \\
$(.622)$ \\
\end{tabular} & $\begin{array}{l}-.168 \\
(-1.036)\end{array}$ & $\begin{array}{l}-.166 \\
(-.685)\end{array}$ & EB & $\begin{array}{l}.531 \\
(.665)\end{array}$ & $\begin{array}{l}.057 \\
(.322)\end{array}$ & $\begin{array}{l}-.152 \\
(-.842)\end{array}$ & $\begin{array}{l}-.242 \\
(-.889)\end{array}$ \\
\hline ESOP & $\begin{array}{l}.146 \\
(-.331)\end{array}$ & $\begin{array}{l}.246 \\
(1.328)\end{array}$ & $\begin{array}{l}.073 \\
(.451)\end{array}$ & $\begin{array}{l}.040 \\
(.265)\end{array}$ & ESOP & $\begin{array}{l}.037 \\
(.066)\end{array}$ & $\begin{array}{l}.073 \\
(-.353)\end{array}$ & $\begin{array}{l}.052 \\
(.683)\end{array}$ & $\begin{array}{l}.075 \\
(.676)\end{array}$ \\
\hline DEBT & $\begin{array}{l}.131 \\
(.084) \\
\end{array}$ & $\begin{array}{l}1.558 \\
(1.805)\end{array}$ & $\begin{array}{l}.411^{\star *} \\
(-3.388) \\
\end{array}$ & $\begin{array}{l}.090 \\
(.212) \\
\end{array}$ & RECEIV & $\begin{array}{l}.647 \\
(.396) \\
\end{array}$ & $\begin{array}{l}1.577 \\
(-1.905) \\
\end{array}$ & $\begin{array}{l}.098 \\
(-.937) \\
\end{array}$ & $\begin{array}{l}.010 \\
(-.042) \\
\end{array}$ \\
\hline INV & $\begin{array}{l}.061 \\
(-.092) \\
\end{array}$ & \begin{tabular}{|l|}
.163 \\
$(.997)$ \\
\end{tabular} & $\begin{array}{l}. \mathbf{1 7 4}^{\star} \\
(1.993)\end{array}$ & $\begin{array}{l}.068 \\
(.596)\end{array}$ & INV & $\begin{array}{l}.081 \\
(.413)\end{array}$ & \begin{tabular}{|l|}
$.456^{\star}$ \\
$(2.277)$ \\
\end{tabular} & \begin{tabular}{|l|}
-.058 \\
$(-.154)$ \\
\end{tabular} & \begin{tabular}{|l|}
-.234 \\
$(-.237)$ \\
\end{tabular} \\
\hline UNEARN & $\begin{array}{l}-.220 \\
(-.459) \\
\end{array}$ & $\begin{array}{l}.153 \\
(.458) \\
\end{array}$ & $\begin{array}{l}112 \\
(.868) \\
\end{array}$ & $\begin{array}{l}.096 \\
(.243) \\
\end{array}$ & DEFER & $\begin{array}{l}.039 \\
(.230) \\
\end{array}$ & \begin{tabular}{|l|}
.142 \\
$(.661)$ \\
\end{tabular} & $\begin{array}{l}.048 \\
(.150) \\
\end{array}$ & $\begin{array}{l}.213 \\
(.282) \\
\end{array}$ \\
\hline DERIV & $\begin{array}{l}.097 \\
(-.211)\end{array}$ & $\begin{array}{l}.649^{\star \star} \\
(-4.044)\end{array}$ & $\begin{array}{l}.100 \\
(-.974)\end{array}$ & $\begin{array}{l}.041 \\
(.163)\end{array}$ & DERIV & $\begin{array}{l}-.145 \\
(-.266)\end{array}$ & \begin{tabular}{|l|}
-.013 \\
$(-.039)$
\end{tabular} & $\begin{array}{l}. \mathbf{4 2 4}^{\star} \\
(-2.378)\end{array}$ & $\begin{array}{l}.202 \\
(-.439)\end{array}$ \\
\hline INVEST & $\begin{array}{l}.278 \\
(-.427)\end{array}$ & \begin{tabular}{|l|}
.007 \\
$(.036)$ \\
\end{tabular} & $\begin{array}{l}.220 \\
(-.635)\end{array}$ & $\begin{array}{l}-.123 \\
(-.227)\end{array}$ & FV & $\begin{array}{l}-.119 \\
(-.578)\end{array}$ & \begin{tabular}{|l|}
$-.442^{* *}$ \\
$(-2.843)$
\end{tabular} & $\begin{array}{l}.005 \\
(.023)\end{array}$ & $\begin{array}{l}.506 \\
(-1.474)\end{array}$ \\
\hline EQUITY & $\begin{array}{l}.065 \\
(.316)\end{array}$ & \begin{tabular}{|l|}
$\mathbf{5 2 4}^{* *}$ \\
$(2.721)$
\end{tabular} & $\begin{array}{l}.122 \\
(-.325)\end{array}$ & $\begin{array}{l}.286 \\
(.285)\end{array}$ & EQUITY & $\begin{array}{l}.019 \\
(.092)\end{array}$ & \begin{tabular}{|l|}
.252 \\
$(.998)$ \\
\end{tabular} & $\begin{array}{l}.093 \\
(-.838)\end{array}$ & $\begin{array}{l}.008 \\
(.061)\end{array}$ \\
\hline INTAN & $\begin{array}{l}.238 \\
(1.309) \\
\end{array}$ & \begin{tabular}{|l|}
.005 \\
$(.051)$ \\
\end{tabular} & \begin{tabular}{|l|}
.029 \\
$(.303)$ \\
\end{tabular} & \begin{tabular}{|l|}
-.042 \\
$(-.267)$ \\
\end{tabular} & INTAN & \begin{tabular}{|l|}
.184 \\
$(1.172)$ \\
\end{tabular} & \begin{tabular}{|l|}
-.489 \\
$(-1.726)$ \\
\end{tabular} & $\begin{array}{l}.009 \\
(.077) \\
\end{array}$ & \begin{tabular}{|l|}
.047 \\
$(.276)$ \\
\end{tabular} \\
\hline ITEMS & $\begin{array}{l}.187 \\
(-.398) \\
\end{array}$ & $\begin{array}{l}.042 \\
(.162) \\
\end{array}$ & $\begin{array}{l}.152 \\
(-.585) \\
\end{array}$ & \begin{tabular}{|l|}
-.073 \\
$(-.562)$ \\
\end{tabular} & LOSSES & \begin{tabular}{|l|}
-.035 \\
$(-.171)$ \\
\end{tabular} & \begin{tabular}{|l|}
-.206 \\
$(-.870)$ \\
\end{tabular} & $\begin{array}{l}.061 \\
(-.635) \\
\end{array}$ & $\begin{array}{l}.001 \\
(-.006) \\
\end{array}$ \\
\hline LOSSES & $\begin{array}{l}.252 \\
(-.997)\end{array}$ & \begin{tabular}{|l|}
.401 \\
$(.975)$
\end{tabular} & $\begin{array}{l}.239^{\star} \\
(1.948)\end{array}$ & $\begin{array}{l}.243 \\
(.972)\end{array}$ & CONV & \begin{tabular}{|l|}
-.035 \\
$(-.187)$
\end{tabular} & $\begin{array}{l}.294 \\
(1.714)\end{array}$ & $\begin{array}{l}.022 \\
(.307)\end{array}$ & $\begin{array}{l}.051 \\
(.441)\end{array}$ \\
\hline RESERV & $\begin{array}{l}-.003 \\
(-.009) \\
\end{array}$ & \begin{tabular}{|l}
.393 \\
$(1.378)$ \\
\end{tabular} & $\begin{array}{l}.399^{\star} \\
(2.481)\end{array}$ & \begin{tabular}{|l|}
$.631^{\star}$ \\
$(1.918)$ \\
\end{tabular} & PROV & \begin{tabular}{|l|}
-.115 \\
$(-.368)$ \\
\end{tabular} & $\begin{array}{l}.140 \\
(.398) \\
\end{array}$ & $\begin{array}{l}.036 \\
(.266)\end{array}$ & $\begin{array}{l}.261 \\
(-1.090) \\
\end{array}$ \\
\hline ACCRUE & $\begin{array}{l}.026 \\
(-.047) \\
\end{array}$ & $\begin{array}{l}.133 \\
(.497) \\
\end{array}$ & \begin{tabular}{|l|}
$-.473^{\star}$ \\
$(-1.991)$ \\
\end{tabular} & \begin{tabular}{|l|}
.262 \\
$(.966)$ \\
\end{tabular} & ACCRUE & $\begin{array}{l}.001 \\
(.009) \\
\end{array}$ & \begin{tabular}{|l|}
.117 \\
$(.923)$ \\
\end{tabular} & \begin{tabular}{|l|}
.054 \\
$(.237)$ \\
\end{tabular} & \begin{tabular}{|l|}
-.071 \\
$(-.232)$ \\
\end{tabular} \\
\hline OTHER & $\begin{array}{l}.100 \\
(.210)\end{array}$ & $\begin{array}{l}.465 \\
(.936)\end{array}$ & $\begin{array}{l}-.149 \\
(-.888)\end{array}$ & $\begin{array}{l}.361 \\
(.846)\end{array}$ & OTHER & $\begin{array}{l}-.128 \\
(-.487)\end{array}$ & $\begin{array}{l}.107 \\
(.507)\end{array}$ & $\begin{array}{l}-.068 \\
(-.244)\end{array}$ & $\begin{array}{l}.076 \\
(.262)\end{array}$ \\
\hline
\end{tabular}

2009 through 2010, the previous answer continues to be correct. This result seems to discourage save for the DTA of DERIV and the DTL of FV as no other variable is statistically significant even twice.

The new DTAs and DTLs data developed for this research do not produce answers that are satisfactory in determining which components of DTAs and DTLs influence stock prices. The relationship between book-tax differences and stock prices shown in general, not specific, terms through the work of researchers discussed in the literature review could have more difficult to discern drivers than they have considered. Many researchers believe that the DTAs would largely be related to increases in stock prices with the DTLs largely related to decreases in stock prices. The tables show combinations of 
components that seem to relate to increases and decreases in stock prices within the DTAs and within the DTLs.

Despite any questions to the contrary, as the literature review shows, this model builds on already established means for testing this category of data. To explain these results, the model could not have sufficient power, or there could be measurement error within the variables that makes it seem that the movements within DTAs and DTLs as related to stock prices are not significant in the expected directions. However, this model has sufficient power for some variables to take on statistical significance and consists of higher quality data than ever before that should result in less measurement error. Thus, the results do have meaning as they seem to indicate that, other than the DTLs of

Table 12

Measuring surprise: regressing changes in price 2006 through 2007 on differences between changes in 2005-06 DTAs and DTLs and changes in 2004-05 DTAs and DTLs

\begin{tabular}{|c|c|c|c|c|c|c|c|c|c|}
\hline $\begin{array}{l}\text { DTAs } \\
\text { Variable }\end{array}$ & 06-07 & 07-08 & 08-09 & $09-10$ & $\begin{array}{l}\text { DTLs } \\
\text { Variable }\end{array}$ & $06-07$ & 07-08 & 08-09 & 09-10 \\
\hline DEPR & $\begin{array}{l}.048 \\
(-.290)\end{array}$ & $\begin{array}{l}-.080 \\
(-.597)\end{array}$ & $\begin{array}{l}.057 \\
(.139)\end{array}$ & $\begin{array}{l}-.052 \\
(-.261)\end{array}$ & DEPR & $\begin{array}{l}.246 \\
(1.151)\end{array}$ & $\begin{array}{l}.839^{\star *} \\
(-4.026)\end{array}$ & \begin{tabular}{|l|}
-.339 \\
$(-1.362)$
\end{tabular} & \begin{tabular}{|l|}
-.336 \\
$(-1.560)$
\end{tabular} \\
\hline EB & $\begin{array}{l}.060 \\
(-.311)\end{array}$ & $\begin{array}{l}.061 \\
(-.349)\end{array}$ & $\begin{array}{l}.050 \\
(.211)\end{array}$ & $\begin{array}{l}.047 \\
(.191)\end{array}$ & EB & \begin{tabular}{|l|}
.655 \\
$(1.037)$
\end{tabular} & \begin{tabular}{|l|}
-.013 \\
$(-.090)$ \\
\end{tabular} & \begin{tabular}{|l|}
.126 \\
$(.479)$ \\
\end{tabular} & \begin{tabular}{|l|}
-.043 \\
$(-.157)$ \\
\end{tabular} \\
\hline ESOP & $\begin{array}{l}-.377 \\
(-1.082)\end{array}$ & $\begin{array}{l}.048 \\
(-.315)\end{array}$ & $\begin{array}{l}.045 \\
(.188)\end{array}$ & $\begin{array}{l}.045 \\
(-.296)\end{array}$ & ESOP & $\begin{array}{l}.338 \\
(-.762)\end{array}$ & \begin{tabular}{|l|}
$.387^{\star}$ \\
$(2.294)$
\end{tabular} & $\begin{array}{l}.029 \\
(-.262)\end{array}$ & \begin{tabular}{|l|}
.017 \\
$(.152)$ \\
\end{tabular} \\
\hline DEBT & $\begin{array}{l}-2.160 \\
(-1.754)\end{array}$ & 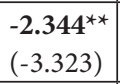 & \begin{tabular}{|l|}
.186 \\
$(1.047)$ \\
\end{tabular} & \begin{tabular}{|l|}
349 \\
$(.819)$ \\
\end{tabular} & RECEIV & $\begin{array}{l}.874 \\
(-.677) \\
\end{array}$ & \begin{tabular}{|l}
$2.108^{* *}$ \\
$(3.117)$
\end{tabular} & \begin{tabular}{|l|}
.211 \\
$(1.384)$
\end{tabular} & $\begin{array}{l}.109 \\
(.449)\end{array}$ \\
\hline INV & $\begin{array}{l}-.281 \\
(-.536) \\
\end{array}$ & \begin{tabular}{|l|}
$-.292^{\star}$ \\
$(-2.189)$ \\
\end{tabular} & $\begin{array}{l}-.094 \\
(-.733) \\
\end{array}$ & $\begin{array}{l}.105 \\
(-.913) \\
\end{array}$ & INV & $\begin{array}{l}.112 \\
(.723) \\
\end{array}$ & $\begin{array}{l}. \mathbf{6 2 6}^{* *} \\
(-3.822) \\
\end{array}$ & \begin{tabular}{|l|}
-.265 \\
$(-.479)$ \\
\end{tabular} & $\begin{array}{l}.070 \\
(.070) \\
\end{array}$ \\
\hline UNEARN & $\begin{array}{l}.730^{\star} \\
(1.928) \\
\end{array}$ & \begin{tabular}{|l|}
-.478 \\
$(-1.747)$ \\
\end{tabular} & \begin{tabular}{|l|}
.041 \\
$(.215)$ \\
\end{tabular} & \begin{tabular}{|l|}
-.248 \\
$(-.627)$ \\
\end{tabular} & DEFER & \begin{tabular}{|l}
$.282^{\star}$ \\
$(2.092)$ \\
\end{tabular} & \begin{tabular}{|l|}
-.323 \\
$(-1.841)$ \\
\end{tabular} & \begin{tabular}{|l|}
.165 \\
$(.354)$ \\
\end{tabular} & \begin{tabular}{|l|}
.824 \\
$(1.085)$ \\
\end{tabular} \\
\hline DERIV & $\begin{array}{l}\mathbf{1 . 5 8 2}^{\star \star} \\
(4.353)\end{array}$ & $\begin{array}{l}.509^{\star \star} \\
(3.883)\end{array}$ & $\begin{array}{l}.012 \\
(.079)\end{array}$ & $\begin{array}{l}-.168 \\
(-.670)\end{array}$ & DERIV & $\begin{array}{l}.142 \\
(.329)\end{array}$ & \begin{tabular}{|l|}
$-.570^{\star}$ \\
$(-2.112)$
\end{tabular} & \begin{tabular}{|l|}
.212 \\
$(.811)$
\end{tabular} & $\begin{array}{l}.434 \\
(-.941)\end{array}$ \\
\hline INVEST & $\begin{array}{l}-.376 \\
(-.731) \\
\end{array}$ & $\begin{array}{l}.223 \\
(-1.308) \\
\end{array}$ & $\begin{array}{l}.240 \\
(-.473) \\
\end{array}$ & $\begin{array}{l}.461 \\
(-.846)\end{array}$ & FV & $\begin{array}{l}.436^{* *} \\
(2.671)\end{array}$ & \begin{tabular}{|l|}
$-.340^{\star *}$ \\
$(-2.674)$ \\
\end{tabular} & \begin{tabular}{|l|}
-.029 \\
$(-.088)$ \\
\end{tabular} & $\begin{array}{l}.527 \\
(-1.529) \\
\end{array}$ \\
\hline EQUITY & $\begin{array}{l}-.005 \\
(-.032)\end{array}$ & $\begin{array}{l}-.703^{* *} \\
(-4.467)\end{array}$ & $\begin{array}{l}-.218 \\
(-.396)\end{array}$ & $\begin{array}{l}.023 \\
(.023)\end{array}$ & EQUITY & $\begin{array}{l}.035 \\
(.218)\end{array}$ & $\begin{array}{l}.229 \\
(1.109)\end{array}$ & \begin{tabular}{|l|}
.174 \\
$(1.066)$
\end{tabular} & $\begin{array}{l}.152 \\
(1.137)\end{array}$ \\
\hline INTAN & $\begin{array}{l}-.172 \\
(-1.195)\end{array}$ & $\begin{array}{l}.004 \\
(.052)\end{array}$ & $\begin{array}{l}.041 \\
(-.297)\end{array}$ & $\begin{array}{l}.110 \\
(-.693)\end{array}$ & INTAN & $\begin{array}{l}.145 \\
(1.166)\end{array}$ & \begin{tabular}{|l|}
$.712^{\star *}$ \\
$(3.076)$
\end{tabular} & $\begin{array}{l}.052 \\
(.296)\end{array}$ & $\begin{array}{l}.195 \\
(1.131)\end{array}$ \\
\hline ITEMS & $\begin{array}{l}.536 \\
(1.442)\end{array}$ & $\begin{array}{l}.403 \\
(1.885)\end{array}$ & $\begin{array}{l}.239 \\
(.631) \\
\end{array}$ & $\begin{array}{l}-106 \\
(-.817) \\
\end{array}$ & LOSSES & $\begin{array}{l}.196 \\
(1.207)\end{array}$ & \begin{tabular}{|l|}
.015 \\
$(-.075)$ \\
\end{tabular} & $\begin{array}{l}.064 \\
(.452) \\
\end{array}$ & \begin{tabular}{|l|}
.007 \\
$(.054)$ \\
\end{tabular} \\
\hline LOSSES & $\begin{array}{l}.204 \\
(1.022)\end{array}$ & $\begin{array}{l}-.942^{* *} \\
(-2.801)\end{array}$ & $\begin{array}{l}.152 \\
(-.846)\end{array}$ & $\begin{array}{l}.109 \\
(.436)\end{array}$ & CONV & $\begin{array}{l}.308 \\
(-2.098)\end{array}$ & $\begin{array}{l}.166 \\
(1.185)\end{array}$ & $\begin{array}{l}.029 \\
(-.282)\end{array}$ & \begin{tabular}{|l|}
.063 \\
$(.547)$
\end{tabular} \\
\hline RESERV & $\begin{array}{l}1.310^{* *} \\
(4.199)\end{array}$ & $\begin{array}{l}-.095 \\
(-.407) \\
\end{array}$ & $\begin{array}{l}-.230 \\
(-.977) \\
\end{array}$ & $\begin{array}{l}.264 \\
(.801) \\
\end{array}$ & PROV & $\begin{array}{l}-.036 \\
(-.145) \\
\end{array}$ & $\begin{array}{l}.320 \\
(1.111) \\
\end{array}$ & $\begin{array}{l}-.039 \\
(-.199) \\
\end{array}$ & $\begin{array}{l}.198 \\
(.824) \\
\end{array}$ \\
\hline ACCRUE & $\begin{array}{l}-.004 \\
(-.010)\end{array}$ & $\begin{array}{l}.042 \\
(-.190)\end{array}$ & $\begin{array}{l}.194 \\
(.558)\end{array}$ & $\begin{array}{l}-.148 \\
(-.543)\end{array}$ & ACCRUE & $\begin{array}{l}.194 \\
(-1.883)\end{array}$ & \begin{tabular}{|l|}
.013 \\
$(.123)$
\end{tabular} & \begin{tabular}{|l|}
-.213 \\
$(-.642)$
\end{tabular} & \begin{tabular}{|l|}
-.037 \\
$(-.120)$
\end{tabular} \\
\hline OTHER & $\begin{array}{l}-.088 \\
(-.233)\end{array}$ & \begin{tabular}{|l|}
408 \\
$(1.007)$ \\
\end{tabular} & \begin{tabular}{|l|}
-110 \\
$(-.448)$ \\
\end{tabular} & \begin{tabular}{|l|}
376 \\
$(.878)$ \\
\end{tabular} & OTHER & \begin{tabular}{|l|}
-.237 \\
$(-1.144)$ \\
\end{tabular} & $\begin{array}{l}.113 \\
(.651)\end{array}$ & \begin{tabular}{|l|}
.277 \\
$(.679)$
\end{tabular} & \begin{tabular}{|l|}
-.140 \\
$(-.478)$
\end{tabular} \\
\hline
\end{tabular}


DEPR and FV and, even for those variables, not in every year, the components of DTAs and DTLs under IFRS are not that statistically significant to determining stock prices.

\section{Conclusions}

As many researchers have already demonstrated the relationship between DTAs and DTLs and stock prices, the logical progression is to determine which specific DTAs and DTLs influence stock prices. This research is the first to hand collect data on DTAs and DTLs to answer this question.

The previous research has encountered measurement error as book-tax differences have required estimation. This hand collection resolves the measurement error issue with 600 company years of FTSE 100 data.

Investors could rely on the DTLs of DEPR (property, plant, and equipment items) and FV (mark-to-market investment adjustments) to ascertain stock price changes. However, many of the DTAs and DTLs do not seem to provide significant influences on stock prices.

This research is the first take on new data. The data developed are incredibly valuable on their own. However, the results seem to show less influence from DTAs and DTLs on stock prices under IFRS than these categories have under US GAAP. As such, these results differ from the researchers' findings discussed in the literature review. Because no research has considered these individual DTA and DTL differences, further comparisons with the literature review or any other pursuits are unavailable.

\section{Implications}

Further research should pursue whether the inherent market emphasis on balance sheets under IFRS over income statements under US GAAP helps explain the less significant influence in general of DTAs and DTLs discovered in this research. With that statement made, there is still reason to believe that investors, creditors, regulators, and other users of IFRS financial statements can discern important stock price trends from DTAs and DTLs disclosures. The emphasis must be on whether the efficient or less than fully efficient market hypothesis governs that particular individual's decision process.

Nevertheless, the current findings could have other significant implications. If DTAs and DTLs have diminished reporting influences on stock prices, the decision in the US on whether to follow through with IFRS implementation could be questioned even more. If investors are driving the change, they could drop the consideration to the extent these results provide them with less value relevant information.

The alternative implication is that US GAAP DTAs and DTLs should be similarly separated into their components to determine how influential US GAAP DTAs and DTLs are. Nevertheless, more study is warranted before these sweeping implications receive any merit. 


\section{References}

1. Accounting Principles Board (1967). Opinion No. 11, Accounting for Income Taxes. - Stamford: APB.

2. Amir, E., Kirschenheiter, M. (1997). The Valuation of Deferred Taxes // Contemporary Accounting Research. No. 14, pp. 597-622. doi: 10.1111/ j.1911-3846.1997.tb00543.x.

3. Amir, E., Sougiannis, T. (1999). Analysts' Interpretation and Investors' Valuation of Tax Carryforwards // Contemporary Accounting Research. No. 16, pp. 1-33. doi: 10.1111/j.1911-3846.1999. tb00572.x.

4. Ayers, B. (1998). Deferred Tax Accounting under SFAS No. 109: An Empirical Investigation of its Incremental Value-Relevance Relative to APB No. $11 / /$ The Accounting Review. No. 73, pp. 195-212.

5. Beaver, W., Dukes, R. (1972). Intraperiod Tax Allocation, Earnings Expectations, and the Behavior of Security Prices // The Accounting Review. No. 47, pp. 320-332.

6. Blouin, J. L., Raedy, J. S., Shackelford, D. (2004). Did Dividends Increase Immediately after the 2003 Reduction in Tax Rates? - NBER Working Paper W10301.

7. Chaney, P., Jeter, D. (1994). The Effect of Deferred Taxes on Security Prices // Journal of Accounting, Auditing, and Finance. No. 9, pp. 91-116.

8. Dhaliwal, D., Trezevant, R., Wilkins, M. (2000). Tests of Deferred Tax Explanation of the Negative Association between the LIFO Reserve and Firm Value // Contemporary Accounting Research.
No. 17, pp. 41-59. doi: 10.1111/j.1911-3846.2000. tb00910.x.

9. Financial Accounting Standards Board (1992). Statement of Financial Accounting Standards 109, Accounting for Income Taxes. - Norwalk: FASB.

10. Graham, J. R., Raedy, J. S., Shackelford, D. (2010). Research in Accounting for Income Taxes. - Working paper, NBER. doi: 10.1561/0500000042.

11. Hanlon, M. (2005). The Persistence and Pricing of Earnings, Accruals, and Cash Flows [where] Firms Have Large Book-Tax Differences // The Accounting Review. No. 80, pp. 137-166.

12. Lev, B., Nissim, D. (2004). Taxable Income, Future Earnings, and Equity Values // The Accounting Review. No. 79, pp. 1039-1074. doi: 10.1.1.163.7380.

13. Lipe, R. (1986). The Information Contained in the Components of Earnings // Journal of Accounting Research. No. 24, pp. 37-64.

14. Rayburn, J. (1986). The Association of Operating Cash Flow and Accruals with Security Returns // Journal of Accounting Research. No. 24, pp. 112133.

15. Thomas, J., Zhang, F. (2007). Tax Income Momentum. - Working paper, Yale University, Department of Accounting.

16. Weber, D. (2009). Do Analysts and Investors Fully Appreciate the Implications of Book-Tax Differences for Future Earnings? // Contemporary Accounting Research. No. 26, pp. 11751206. doi: 10.1506/car.26.4.7.

The paper submitted: April 06, 2013 Prepared for publication: June 1, 2013

\section{Kevin A. DIEHL}

\section{LAIKINIEJI FINANSINIO IR MOKESTINIO PELNO SKIRTUMAI PAGAL TFAS} S a n t r a u a

Daugeliu atvejų atidètojo pelno mokesčio turto (APMT) ir atidètojo pelno mokesčio issipareigojimų (APMİ) tyrimai atskleidè galimybę tiksliau prognozuoti ateities akcijų kainų pokyčius, lyginant su prognozemis, kurios remiasi pelno, tenkančio vienai akcijai, arba pinigų srautų rodikliais. Remiantis šiais atvejais, investuotojai, kreditoriai, reguliuojančios institucijos ir kiti, besinaudojantys finansinių ataskaitų duomenimis, privalo suprasti, kaip, remiantis APMT ir APMI, galima prognozuoti akcijų kainų pokyčius. Nepaisant reikšmingų esamų tyrimų, kurie nagrinejo pavienių ar bendrų APMT ir APMI kategorijų poveikio akcijų kainoms atvejus, tyrimų, kurie apimtų visas kategorijas vienu metu, nèra. Be viso to, nè vienas esamas tyrimas neparodè sąryšio tarp APMT ir APMİ bei akcijų kainos, 
remiantis TFAS. Tad, šiuo tyrimu siekiama išskirti, kurie specifiniai skirtumai tarp laikino ataskaitinio ir apmokestinamo pelno (finansinio ir mokestinio pelno skirtumai - angl. Book-tax differences), suteikia galimybę prognozuoti akcijų kainas, remiantis TFAS. Dauguma šalių yra prièmusios TFAS, tačiau žinių apie finansinio ir mokestinio pelno skirtumus, remiantis šiais standartais, trūksta. Šis tyrimas yra ypatingai svarbus toms šalims, kurios nesinaudoja TFAS (pvz. JAV), nes remiantis juo, galima informuoti reguliuojančias ir îstatymus leidžiančias insitucijas apie finansinio ir mokestinio pelno skirtumus, atsirandančius dèl TFAS, tam, kad būtu galima palyginti ir interpretuoti įvairius rodiklius prie skirtingų apskaitos standartų.

Finansinio ir mokestinio pelno skirtumai tyrime analizuojami remiantis FTSE 100 metiniais duomenimis nuo 2004 iki $2010 \mathrm{~m}$. Naudojantis koreliacine ir logistine regresijos analize tiriamas šiu kintamųjų reikšmingumas.

Iki šiol nebuvo tirta JAV esančiu APMT ir APMI prie GAAP standartų, aiškinantis, kurios APMT ir APMI kategorijos yra reikšmingesnès už kitas. Tam itakos galèjo turèti skaitmeninès informacijos stoka. Pirmuoju iššukiu, tiriant finansinio ir mokestinio pelno skirtumų ịtaką, tampa faktas, kad mokesčiu paaiškinimai apima tik kai kuriuos laikinus mokesčiu skirtumus. Tyrimas nagrinèja informacines išnašas apie laikinus mokestinius skirtumus 2004 - 2010 m. laikotarpiu. Tai leidžia pagrịsti akcijų grąžos su specifiniais finansinio ir mokestinio pelno skirtumais tyrimą.

Finansinio ir mokestinio pelno skirtumų reikšmingumo tyrimas yra svarbus, nes šiais mokesčiais siekiama suvienodinti finansines ataskaitas ir mokesčių grąžą. Dauguma ịmonių veiklų vienu ar kitu laikotarpiu remiasi APMT ir APMI. Tad svarbiausia išsiaiškinti, kurie laikini skirtumai turi didžiausią įtaką TFAS kontekste.

Kai kurie finansinio ir mokestinio pelno skirtumai suteikia informacijos, rodančios APMT ir APMI reikšmingumą akcijų kainoms. Dèl to, kad ne visi laikini finansinio ir mokestinio pelno skirtumai suteikia tokią informaciją, kai kurie mokslininkai gali atmesti bendrą nuostatą, jog apskaitos mokesčių skirtumai suteikia svarbią informaciją investuotojams, nustatant akcijų kainas. 